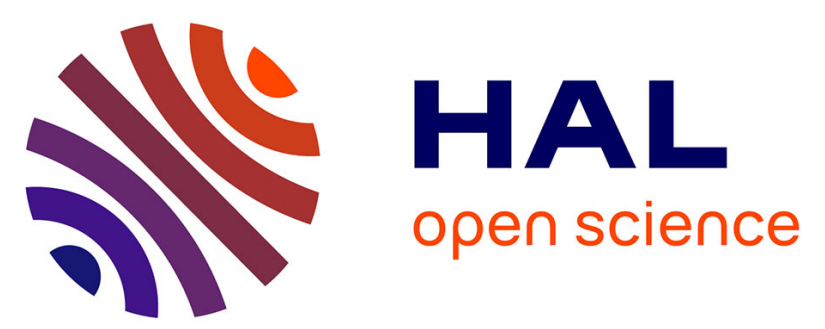

\title{
Neuropilin 1 and Neuropilin 2 gene invalidation or pharmacological inhibition reveals their relevance for the treatment of metastatic renal cell carcinoma
}

Aurore Dumond, Etienne Brachet, Jérôme Durivault, Valérie Vial, Anna

Puszko, Yves Lepelletier, Christopher Montemagno, Marina

Pagnuzzi-Boncompagni, Olivier Hermine, Christiane Garbay, et al.

\section{To cite this version:}

Aurore Dumond, Etienne Brachet, Jérôme Durivault, Valérie Vial, Anna Puszko, et al.. Neuropilin 1 and Neuropilin 2 gene invalidation or pharmacological inhibition reveals their relevance for the treatment of metastatic renal cell carcinoma. Journal of experimental \& clinical cancer research, 2021, 40, pp.33. 10.1186/s13046-021-01832-x . hal-03132345

\section{HAL Id: hal-03132345 \\ https://hal.science/hal-03132345}

Submitted on 29 Nov 2021

HAL is a multi-disciplinary open access archive for the deposit and dissemination of scientific research documents, whether they are published or not. The documents may come from teaching and research institutions in France or abroad, or from public or private research centers.
L'archive ouverte pluridisciplinaire HAL, est destinée au dépôt et à la diffusion de documents scientifiques de niveau recherche, publiés ou non, émanant des établissements d'enseignement et de recherche français ou étrangers, des laboratoires publics ou privés. 


\title{
Neuropilin 1 and Neuropilin 2 gene invalidation or pharmacological inhibition reveals their relevance for the treatment of metastatic renal cell carcinoma
}

\author{
Aurore Dumond ${ }^{1,2}$, Etienne Brachet ${ }^{3}$, Jérôme Durivault ${ }^{1,2}$, Valérie Vial ${ }^{1,2}$, Anna K. Puszko ${ }^{4}$, Yves Lepelletier ${ }^{5,6}$, \\ Christopher Montemagno ${ }^{1,2}$, Marina Pagnuzzi-Boncompagni ${ }^{1,2}$, Olivier Hermine ${ }^{5,6}$, Christiane Garbay ${ }^{7}$, \\ Nathalie Lagarde ${ }^{8}$, Matthieu Montes ${ }^{8}$, Luc Demange ${ }^{3,9}$, Renaud Grépin ${ }^{1,2}$ and Gilles Pagès ${ }^{1,10,2^{*}}$ (D)
}

\begin{abstract}
Background: Despite the improvement of relapse-free survival mediated by anti-angiogenic drugs like sunitinib (Sutent ${ }^{\oplus}$ ), or by combinations of anti-angiogenic drugs with immunotherapy, metastatic clear cell Renal Cell Carcinoma (mccRCC) remain incurable. Hence, new relevant treatments are urgently needed. The VEGFs coreceptors, Neuropilins 1, 2 (NRP1, 2) are expressed on several tumor cells including ccRCC. We analyzed the role of the VEGFs/NRPs signaling in CCRCC aggressiveness and evaluated the relevance to target this pathway.

Methods: We correlated the NRP1, 2 levels to patients' survival using online available data base. Human and mouse cCRCC cells were knocked-out for the NRP1 and NRP2 genes by a CRISPR/Cas9 method. The number of metabolically active cells was evaluated by XTT assays. Migration ability was determined by wound closure experiments and invasion ability by using Boyden chamber coated with collagen. Production of VEGFA and VEGFC was evaluated by ELISA. Experimental cCRCC were generated in immuno-competent/deficient mice. The effects of a competitive inhibitor of NRP1 2, NRPa-308, was tested in vitro and in vivo with the above-mentioned tests and on experimental ccRCC. NRPa308 docking was performed on both NRPs.

(Continued on next page)
\end{abstract}

\footnotetext{
*Correspondence: Gilles.Pages@unice.fr; gpages@unice.fr

${ }^{1}$ Scientific Center of Monaco, Biomedical Department, 8 Quai Antoine ler, MC-98000 Monaco, Principality of Monaco

${ }^{10}$ University Cote d'Azur (UCA), Institute for research on cancer and aging of Nice, CNRS UMR 7284; INSERM U1081, Centre Antoine Lacassagne, Nice, France

Full list of author information is available at the end of the article
}

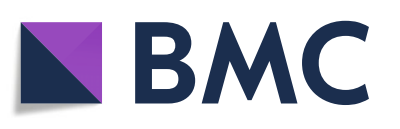

(0) The Author(s). 2021 Open Access This article is licensed under a Creative Commons Attribution 4.0 International License, which permits use, sharing, adaptation, distribution and reproduction in any medium or format, as long as you give appropriate credit to the original author(s) and the source, provide a link to the Creative Commons licence, and indicate if changes were made. The images or other third party material in this article are included in the article's Creative Commons licence, unless indicated otherwise in a credit line to the material. If material is not included in the article's Creative Commons licence and your intended use is not permitted by statutory regulation or exceeds the permitted use, you will need to obtain permission directly from the copyright holder. To view a copy of this licence, visit http://creativecommons.org/licenses/by/4.0/. The Creative Commons Public Domain Dedication waiver (http://creativecommons.org/publicdomain/zero/1.0/) applies to the data made available in this article, unless otherwise stated in a credit line to the data. 
(Continued from previous page)

Results: Knock-out of the NRP1 and NRP2 genes inhibited cell metabolism and migration and stimulated the expression of VEGFA or VEGFC, respectively. NRPa-308 presented a higher affinity for NRP2 than for NRP1. It decreased cell metabolism and migration/invasion more efficiently than sunitinib and the commercially available NRP inhibitor EG00229. NRPa-308 presented a robust inhibition of experimental ccRCC growth in immunocompetent and immunodeficient mice. Such inhibition was associated with decreased expression of several pro-tumoral factors. Analysis of the TCGA database showed that the NRP2 pathway, more than the NRP1 pathway correlates with tumor aggressiveness only in metastatic patients.

Conclusions: Our study strongly suggests that inhibiting NRPs is a relevant treatment for mccRCC patients in therapeutic impasses and NRPa-308 represents a relevant hit.

Keywords: ccRCC, Neuropilins, Oncology, Immunology, Cancer

\section{Background}

Clear cell Renal Cell Carcinoma (ccRCC) is the most common form of kidney cancers $[1,2]$. If non-metastatic, surgery remains the best treatment. However, metastatic ccRCC (mccRCC) are chemo- and radio-resistant. Most of ccRCC are inactivated for the von Hippel-Lindau gene ( $V H L)$, leading to Hypoxia Inducible Factor- 1 and 2 alpha (HIF-1, $2 \alpha$ ) stability [3]. HIFs are key players in tumor aggressiveness and drug resistance particularly through Vascular Endothelial Growth Factor-A (VEGFA)-dependent angiogenesis and VEGFC-dependent lymphangiogenesis [3].

VEGFA exerts its activity through its receptors VEGFR1 and -2 and its coreceptor Neuropilin 1 (NRP1) and VEGFC through VEGFR2 and -3 and Neuropilin 2 (NRP2) [4].

Thus, the current reference treatments target the VEGFs/VEGFRs dependent angiogenesis. Two strategies are currently used: i) antibodies or decoy receptors targeting VEGFA such as bevacizumab (Avastin ${ }^{\circledR}$ ) or aflibercept (Zaltrap ${ }^{\oplus}$ ) and ii) tyrosine-kinase inhibitors (TKi) targeting the kinase domain of VEGFs receptors such as sunitinib (Sutent ${ }^{\oplus}$ ) [5].

The efficacy of the current first-line treatment sunitinib varies from a patient to another with a median survival varying from few months to few years [6]. This transient effect is in part explained by the development of an alternative lymphatic network dependent on the production of VEGFC by tumor cells or cells of the microenvironment in reaction to the stress induced by the drug [7]. Hence, the destruction of the vascular network leads to the development of an alternative network favoring metastatic dissemination. These results highlight the need to develop alternative therapeutic strategies for the treatment of mccRCC especially at relapse on anti-angiogenic drugs.

To prevent redundancy of the vascular and lymphatic networks in response to the reference treatments, the VEGFA/VEGFRs coreceptors, the NRPs, may represent relevant targets in oncology [8-10]. NRPs, 120-130 kDa transmembrane glycoproteins, were initially described as mediators of neuronal guidance. NRP1 and NRP2 share $44 \%$ amino acid sequence identity and close domain structures. They form ternary complexes with VEGFs and their tyrosine kinase associated domains and represent key actors of the pro-angiogenic and prolymphangiogenic signaling pathways. NRPs are also expressed on immune cells where they exert an activation or a repression of the immune response [11]. Moreover, NRPs overexpression in cancer cells is correlated to a high metastatic potential and to a poor prognosis [12]. Down-regulation of NRP1 by shRNA in ccRCC cells decreases migration, invasion and experimental human tumor growth [10], while NRP2 down-regulation results in decreased tumor cell extravasation in the lymphatic network and reduced cell metastatic dissemination in immunodeficient models [9]. Thus, targeting NRPs in ccRCC appears as a relevant therapeutic strategy. To this end, we developed a NRPs inhibitor, NRPa308. It exerts anti-angiogenic and anti-proliferative effects, and prevents the growth of experimental models of highly aggressive triple negative breast cancers $[8,13]$.

The aim of this study was to validate the relevance of NRPs targeting in models of ccRCC generated in the presence of an active immune system. Although immunotherapy showed promising results in mccRCC, only $30 \%$ of patients beneficiate of the treatment [14]. We further determined the antitumor effect of NRPa308 on experimental ccRCC and compared its efficacy to the referent treatment sunitinib through in vitro and in vivo approaches.

\section{Materials and methods Reagents}

NRPa-308 has been synthesized at the University of Paris (Luc Demange's team). Sunitinib was purchased from Selleckem or from residual materials given to patients (Center Antoine Lacassagne, Nice, France) and prepared as a $2.5 \mathrm{mmol} / \mathrm{L}$ stock solution in dimethyl sulfoxide (Sigma, 472,301) and stored at $-20^{\circ} \mathrm{C}$. EG00229 was purchased from Tocris. 


\section{Cell lines}

786-O, A498 and RENCA cell lines were purchased from the American Tissue Culture Collection (ATCC). They were cultured as indicated by ATCC and as already described [15].

\section{Genomic disruption of Neuropilins using CRISPR-CAS9 and ShRNA}

786-O or RENCA cells were transfected with PX458 plasmids containing CRISPR-Cas9 targeting region of the second exon of the NRP1 or of the NRP2 gene using $\mathrm{NaCl}$ and PEI. Control cells were obtained by transfecting the empty plasmid. The sgRNA sequence used to target the human NRP1 gene was: 5'-CGGGTACCTTACATCTCC TG-3'; the sgRNA sequence targeting the human NRP2 gene was: 5' - TTCAAACGACCTCCGCACGG-3'. The sgRNA sequence used to target the mouse $n r p 1$ gene was: 5' - GCAAGACTCGAATCCTCCCGG - 3'; the sgRNA sequence targeting the mouse $n r p 2$ gene was: $5^{\prime}$ - GTGG ATCAAATAGTAACGTG - $3^{\prime}$. As the PX458 plasmid contains GFP, cells were first sorted using flow cytometry to obtain cells containing the CRISPR-Cas9 and followed by clonal selection and screening. Sequencing of human genomic DNA to confirm the mutations leading to human NRP1 or NRP2 invalidation was performed using the following primers: Forward NRP1 5'- CACGAAGGAC TTACGGGG-3' and Reverse NRP1 5' - AGACAGGCGT GACCAGTAG-3', and Forward NRP2 5' - TGAGCCGG AATAATCTCTTCCAC-3' and Reverse NRP2 5' - GGTG CTTACTTGCAGTCGTG-3'. Sequencing of mouse genomic DNA was performed with the following oligonucleotides: Forward nrp1 5'- ACAGGGCCCGAATGTTCTC $-3^{\prime}$ and Reverse nrp1 5' - TTCACAGACTCCATTGCC TG $-3{ }^{\prime}$, and Forward nrp2 5' - TTTACATCAAGGCA TTGGCAG - 3' and Reverse nrp2 5'- GTGGAAGTTA CGGGATCGTATAGTC -3 '.

\section{Quantitative real-time PCR (qPCR) experiments}

One microgram of total RNA was used for the reverse transcription, using the QuantiTect Reverse Transcription Kit (Qiagen), with blend of oligo (dT) and random primers to prime first strand synthesis. SYBR Master Mix Plus (Eurogentec) was used for quantitative realtime PCR (qPCR). The mRNA level was normalized to 36B4 mRNA. For oligo sequences, see Table S1.

\section{Protein level measurement by flow cytometry analysis (FACS)}

Knocked-out (KO) cells were incubated for one hour with primary antibodies: i) Polyclonal sheep IgG Human anti-NRP1 antibody (AF3870 (sheep); R\&D systems); ii) Polyclonal goat IgG Human/Mouse/Rat anti-NRP2 antibody (AF2215 (goat); R\&D systems). After washing with cold PBS, the cells were incubated for $30 \mathrm{~min}$ with the secondary antibodies: i) Donkey Anti-Sheep IgG H\&L, Alexa Fluor 594 (Abcam); ii) Goat anti-Human IgG $(\mathrm{H}+\mathrm{L})$ Cross-Adsorbed Secondary Antibody, Alexa Fluor 488 (Thermo Fisher). The cells were then washed with cold PBS and resuspended in the FACS medium (cold PBS + $2.5 \mathrm{mM}$ EDTA). NRP1 and NRP2 level expression were determined using a fluorescence-activated cell sorter (FACS Melody BD Biosciences) with a 488 $\mathrm{nm}$ and a $594 \mathrm{~nm}$ laser beam.

\section{Measurement of cytokines}

CXCL8 cytokines and VEGFA were detected by using PeproTech ELISA kits according to the manufacturer's indication as already described [16]. VEGFC and CXCL5 were measured using R\&D systems ELISA kits according to the manufacturer recommendations.

\section{Measurement of cell migration velocity and invasive ability}

At confluency, a wound was created on the cell monolayer and its width was measured every hour for $10 \mathrm{~h}$ to determine the migration velocity. At the end of the experiment, the cells were counted to verify if cell death or proliferation had not influenced the wound closure. To evaluate invasion, cells were seeded onto the upper side of the collagen-coated filters of Boyden chambers containing polycarbonate membranes $(8-\mu \mathrm{m}$ pores, Transwell; Corning, Sigma). Cell invasion was followed for 24 $\mathrm{h}$ at $37^{\circ} \mathrm{C}$ in $5 \% \mathrm{CO} 2$. Invasive cells on the lower membrane surface were fixed in $3 \%$ paraformaldehyde, stained with $0.4 \%$ crystal violet. Cells were counted using the Image J software.

\section{Competitive NRP1/2 VEGFA/VEGFC binding assay}

The flat bottom surface of a 96-well plate was coated with $100 \mu \mathrm{L}$ ( $200 \mathrm{ng} /$ well) recombinant human NRP1 or NRP2 and incubated overnight at $4{ }^{\circ} \mathrm{C}$. Non-specific binding was blocked by the incubation with $0.5 \% \mathrm{BSA}$ in PBS. $50 \mu \mathrm{L}$ of NRPa-308 dissolved in range concentrations and $50 \mu \mathrm{L}(400 \mathrm{ng} / \mathrm{mL})$ of human (bt)-VEGFA or VEGFC in PBS containing $4 \mu \mathrm{g} / \mathrm{mL}$ of heparin were mixed. After two hours incubation at room temperature, the (bt)-VEGFA plate was washed and treated with streptavidin-horseradish peroxidase (HRP) conjugate in PBS (1:8000). The VEGFC plate was incubated with (bt)anti-VEGFC for one hour and then revealed using HRP conjugate. Luminescence was quantified immediately after addition of $100 \mu \mathrm{L}$ chemiluminescent substrate. In a positive control, only (bt)-VEGFA or VEGFC was present in wells, while, in negative control (NS), wells were not coated with NRP1 or NRP2. Percentages of inhibition were calculated by the following formula: $100 \%$ - $[[(\mathrm{S}-\mathrm{NS}) /(\mathrm{P}-\mathrm{NS})] \cdot 100 \%]$, where $\mathrm{S}$ is the signal intensity measured, NS is the signal measured in negative 
control, and $\mathrm{P}$ is the signal measured in positive control. Presented data are the mean \pm SEM of two or three independent experiments, each performed in triplicate.

\section{Docking study}

NRP1 (PDB ID: 6FMF) and NRP2 (PDB ID: 5DN2) structures were retrieved from the Protein Data Bank (PDB) [17]. The NRP2 structure was aligned with the NRP1 structure and both structures were prepared using MGL tools (https:// www.ncbi.nlm.nih.gov/pubmed/19399780. (Accessed: 1st February 2019). Three-dimensional conformations of NRPa308 were generated using iCon, the LigandScout v.4.3 conformer generator [18] (defaults settings of the BEST option were used, except for the maximum number of conformations generated that was set to 50 instead of 25). Protein - ligand docking of compound NRPa-308 into the NRP1 and NRP2 structures was performed using AutoDock Vina v.1.1.2 [19]. The $x, y, z$ grid centre coordinates used are $12.045,21.518,15.783$ and the size of the search space was set to $20 \AA \times 20 \AA \times 20 \AA$. Only the pose associated with the best score was considered for each run.

\section{Tumor xenograft formation, size evaluation and treatment}

786-O cells expressing luciferase (Luc 1 ) or RENCA cells expressing luciferase (Luc 2) were injected subcutaneously into the flanks of 5 weeks old nude female mice or Balb-C mice. Treatment by NRPa-308 in carboxymethyl cellulose was carried out by oral gavage 3 days a week; the control group was treated with carboxymethyl cellulose. Tumors measurements were carried out once a week with a caliper and by luciferase measurements with IVIS chamber as previously described [20]. All animal procedures were performed according to the Monaco animal experimentation guidelines in strict accordance with the recommendations in the Guide for the Care and Use of Laboratory Animals. Our experiments were approved by our internal ethic committee.

\section{Immuno-fluorescence}

Tumor sections $(5 \mu \mathrm{m}$ cryostat sections) were fixed in $4 \%$ paraformaldehyde for $20 \mathrm{~min}$ at room temperature and blocked in $1 \%$ donkey serum in tris-buffered saline (TBS) for $2 \mathrm{~h}$. Sections were then incubated overnight with anti-rabbit LYVE-1 polyclonal (Ab14917, 1:200; Abcam) or rat monoclonal anti-mouse CD31 (clone MEC 13.3, 1:1000; BD Pharmingen) and monoclonal anti- mouse $\alpha$-smooth muscle actin ( $\alpha$ SMA A2547, 1: 1000; Sigma) antibodies. Preparations were mounted and analyzed with a Leica microscope, and counted at a $10 x$ magnification.

\section{Patients online data}

Normalized RNA sequencing (RNA-Seq) data of The Cancer Genome Atlas (TCGA) were downloaded from cBioportal (www.cbioportal.org, TCGA Provisional; RNA-Seq V2). Data were available for 534 RCC tumor samples or from 1020 different cell lines. The results published here are in whole or in part based upon data generated by the TCGA Research Network: http:// cancergenome.nih.gov/ [21, 22].

\section{Statistical analysis}

Statistical significance and $P$ values were determined with the two-tailed t-test. The Kaplan-Meier method was used to produce survival curves and analyses of censored data were performed using the log-rank test.

\section{Results}

NRP1 or NRP2 gene invalidation resulted in inhibition of cell proliferation and migration

According to the papers of Cao $\mathrm{Y}$ et al $[9,10]$, neither $N R P 1$ or NRP2 knock-down impacted cell proliferation (NRP1/NRP2) and migration (NRP2). These results were surprising since they described that NRP1 knock-down decreased the AKT activity, a major pathway involved in cell proliferation/survival. Moreover, the NRPs-mediated signaling pathways were associated with cell proliferation and migration in several cancers [23]. We first tried to confirm the results of Cao $\mathrm{Y}$ et al particularly on cell proliferation and migration by using the same shRNA and two other independent shRNA through lentiviral infection. The knock-down levels that we obtained were comparable to those described by Cao Y et al (Fig. S1AB). NRP1 knock-down decreased NRP2 expression, an observation that was not described in the Cao $\mathrm{Y}$ et al papers. This observation, confirmed with two independent shRNA, eliminates an unspecific effect, and suggests a crosstalk between the NRP1 and NRP2 signaling pathways (Fig. S1A-B). Whereas Cao Y et al did not detect modifications of cell proliferation and migration at $24 \mathrm{~h}$, we observed a small but significant inhibition of cell metabolic activity for the shNRP1 cells at $72 \mathrm{~h}$ and a surprising increased cell metabolic activity for the shNRP2 cells (Fig. S1C). The migration velocity was also significantly inhibited for shNRP1 and shNRP2 cells (Fig. S1D). The privileged NRP1 ligand VEGFA and the privileged NRP2 ligand VEGFC were not affected by the knockdowns (Fig. S1E). These differences with the results of Cao $\mathrm{Y}$ et al incited us to decipher the role of NRP1 and NRP2 by knocking-out (KO) their genes by the CRISPR/Cas9 method in human (786-O) and mouse (RENCA) ccRCC cells. Two independent KO clones for NRP1 and NRP2 genes were obtained for 786-O (Fig. 1a-b), and one KO clone for NRP1 and NRP2 genes for RENCA (Fig. S2A) cells. Specific NRP1 and NRP2 
A

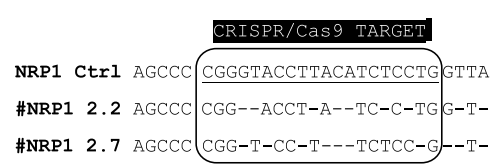

C NRP1 mRNA level NRP2 mRNA level

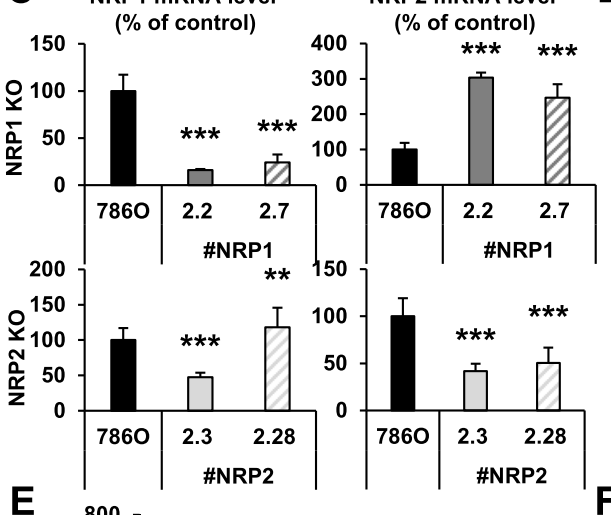

$\mathbf{E}$
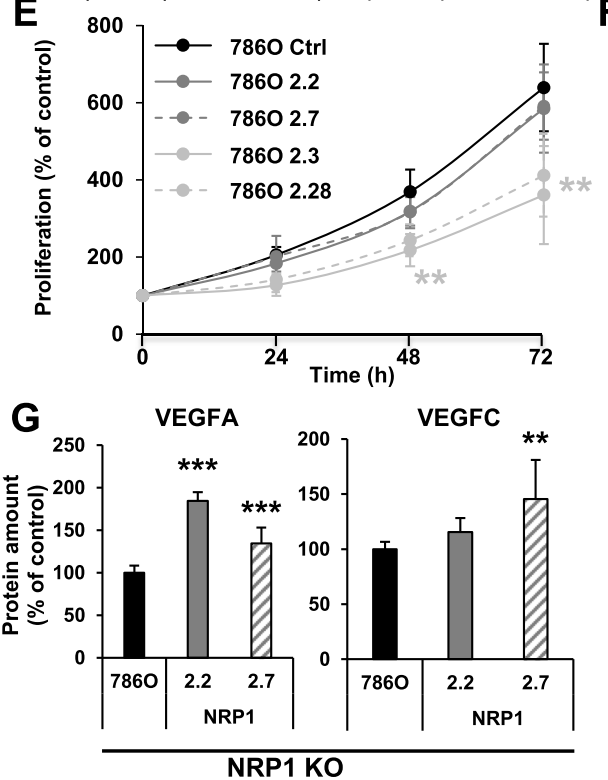

B
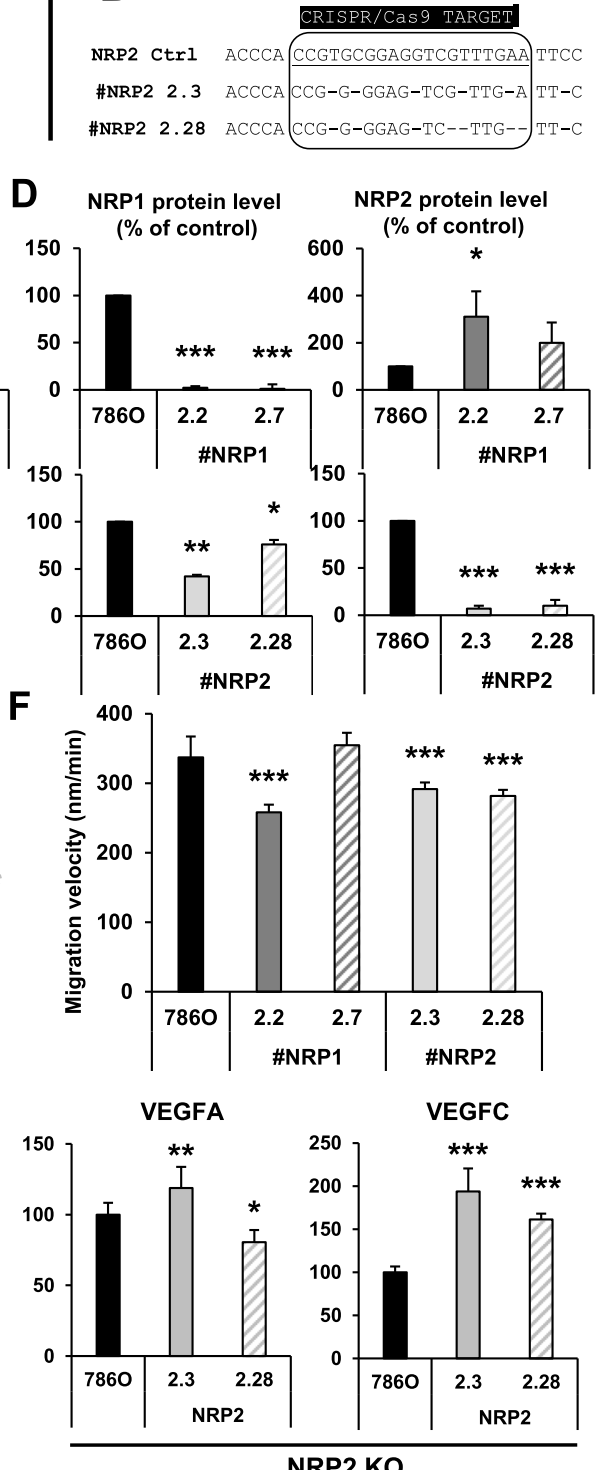

NRP2 KO

Fig. 1 NRP1 or NRP2 gene invalidation results in inhibition of cell proliferation and migration. a The locus of the NRP1 gene was sequenced in control (NRP1 Ctrl) and in two independent clones (\#NRP1 2.2 and \#NRP1 2.7) KO for NRP1. b The locus of NRP2 was sequenced in control (NRP2 Ctrl) and in two independent clones (\#NRP2 2.3 and \#NRP1 2.28) KO for NRP2. c NRP1 and NRP2 mRNA levels were tested by qPCR in control (7860), in two independent clones (\#NRP1 2.2 and 2.7) KO for NRP1, and in two independent clones (\#NRP2 2.3 and 2.28) KO for NRP2. d NRP1 and NRP2 protein levels were evaluated by flow cytometry in control (7860), in two independent clones (\#NRP1 2.2 and 2.7) KO for NRP1, and in two independent clones (\#NRP2 2.3 and 2.28) KO for NRP2. e The proliferation of NRP1 and NRP2 KO cells were tested by counting the cells at the indicated time points. $\mathbf{f}$ The migration of NRPS KO cells was determined in scratch assays by measuring the time of wound closure. $\mathbf{g}$ VEGFA and VEGFC expression was tested in control (Ctrl) and KO clones by ELISA. ${ }^{*} p<0.05$; ${ }^{* *} p<0.01$; ${ }^{* *} p<0.001$

mRNA levels were very low and protein levels were almost undetectable in the $\mathrm{KO}$ clones (Fig. 1c-d and Fig. $\mathrm{S} 2 \mathrm{~A})$. However, NRP1 KO tends to increase NRP2 levels whereas NRP2 $\mathrm{KO}$ tends to decrease NRP1 levels in 786-O cells at the mRNA and protein levels (Fig. 1d). A completely different guide RNAs for NRP1 and NRP2 [24] genes that were positioned adequately for the presence of the PAM sequence [25] excluded also unspecific effects. Although the trend in decreased NRP1 levels were consistently observed in NRP2 KO RENCA cells, the NRP1 KO resulted in decreased expression of NRP2 in RENCA cells (Fig. S2A). We obtained opposite results between $\mathrm{KO}$ and down-regulation of NRP1 by shRNA for NRP2 expression in 786-O cells (NRP1-directed shRNA decreased NRP2 levels whereas the KO tends to induce NRP2 expression). All these results, recapitulated 
in Tables S2, were consistent in 786-O and RENCA cells with down-regulation or $\mathrm{KO}$. They suggest a fine-tuned crosstalk between the NRP1 and the NRP2 signaling, which mediates an equilibrated expression of each protein compatible with cell metabolic activity/survival. In 786-O cells, NRP1 KO moderately impacted cell metabolic activity while NRP2 KO decreased it more importantly (Fig. 1e). These results were consistent for NRP1 down-regulation and $\mathrm{KO}$ whereas down-regulation of NRP2 stimulated and KO inhibited cell metabolic activity (Fig. 1e and Fig. S1D). A moderate but still nonsignificant inhibition of cell metabolic activity was also observed in RENCA NRP1 KO cells. NRP2 KO consistently inhibited RENCA cell metabolic activity (Fig. S2B). Except for one NRP1 KO clone (see discussion), NRPs' $\mathrm{KO}$ decreased the migration velocity of $786-\mathrm{O}$ cells which is consistent with the results obtained by downregulating NRP1 and NRP2 (Fig. 1f and Fig. S1C). Since the NRPs' signaling depends on stimulation by their ligands VEGFA and VEGFC, we tested their expression in the $\mathrm{KO}$ cells. In the 786-O model, NRP1 KO and NRP2 $\mathrm{KO}$ resulted in increased expression of their major ligands VEGFA and VEGFC respectively (Fig. 1g). Inconsistent increase in VEGFC (NRP1 KO) or VEGFA (NRP2 $\mathrm{KO})$ has to be paralleled with variable up- or downregulation of NRP2 or NRP1 which reflects a clonal specificity (Fig. 1c-d). In the RENCA model, expression of VEGFA was consistently decreased in NRP1 and NRP2 KO cells and VEGFC was down-regulated only in NRP2 KO cells (Fig. S2C). Table S3 recapitulates VEGFA and VEGFC in the different model cell lines. These results suggest the maintenance of a steady state level of autocrine loops involving the respective NRP1/VEGFA and NRP2/VEGFC signaling pathways. This equilibrium varies from a model to another and is compensated, at least in the human model, by increased expression of the NRPs' ligands.

NRPs KO in tumor cells inhibited experimental RCC growth in immunocompetent and immunodeficient mice Considering the relevance of immune checkpoint inhibitors in the treatment of mccRCC [26], we deciphered the specific role of NRPs expressed by tumor cells on the growth of experimental tumors in immunodeficient and immunocompetent mice. For that purpose, we compared the growth of experimental tumors generated with 786-O $\mathrm{KO}$ cells in nude mice and generated by RENCA KO cells grafted in nude and syngenic BalbC mice. The NRP1 and NRP2 786-O KO clones generated smaller tumors in nude mice as compared to the controls (Fig. S3). This result is consistent with the NRPs-dependent cell metabolic activity in-vitro. Invalidation of NRP1 or NRP2 in RENCA cells delayed tumor incidence (percentage of mice with a tumor) as compared to the control group, in nude mice
(Fig. 2a). Although, NRP KO cells generated tumors in some nude mice, their volumes remained very small as compared to control tumors (Fig. S4). Moreover, injection of the same cells in immunocompetent mice did not generate tumors (Fig. 2b). These results strongly suggest that, in addition to the intrinsic effects of NRPs on tumor cell metabolic activity, their expression on tumor cells inhibits the anti-tumor immune system.

The NRP inhibitor NRPa-308 inhibits ccRCC cell metabolic activity more efficiently than sunitinib and EG00229

The strong impact on tumor growth mediated by invalidation of NRPS encouraged us to test the relevance of the NRPs' pharmacological inhibitor NRPa-308 on different parameters characterizing ccRCC cells (786-O and A498) aggressiveness in comparison to its effect on normal dermal fibroblasts (HDF). The NRPa-308 effects were compared to those of the reference treatment for ccRCC, sunitinib, and to the commercially available NRPs' inhibitor EG00229. EG00229 was poorly efficient in inhibiting the metabolic activity of ccRCC cell lines (5 and 30\% inhibition respectively for 786-O and A498 cells at the highest dose $2 \mu \mathrm{M}$, Fig. 3a-b). Sunitinib inhibited ccRCC cell metabolic activity more efficiently especially in 786-O cells as compared to A498 cells (40\% versus $30 \%$ for the highest dose). $\mathrm{IC}_{50}$ values were lower for NRPa-308 as compared to sunitinib suggesting its higher efficacy on cell metabolic activity. Moreover, the $\mathrm{IC}_{50}$ of NRPa-308 was higher in normal cells (HDF) as compared to tumor cells (Fig. 3c). We then calculated the selectivity index (SI) to evaluate the toxicity on normal tissues. The $\mathrm{IC}_{50}$ of normal cells (HDF) served as the reference value. The SI was below 1 which indicates that NRPa-308 is more efficient on tumor cells and that its general toxicity is low. The SI of NRPa-308 was below those of sunitinib suggesting a higher efficacy and a lower toxicity of NRPa-308 (Fig. 3d).

\section{NRPa-308 exerts a wide range anti-metabolic activity on primary ccRCC cells}

Resistance to the current treatments especially to sunitinib is a real concern [7]. We previously generated sunitinib-resistant 786-O cells (786R) by chronic exposure to the drug [27]. NRPa-308 had no effect on these cells $\left(\mathrm{IC}_{50}>2 \mu \mathrm{M}\right)$ as compared to the parental cells. 786R cells presented a four-fold and a nine-fold reduction of the NRP1 and NRP2 mRNA levels (Fig. 4a-b). These results are compatible with a strong dependence on cell proliferation/survival mediated by the VEGFA/ NRP1 and VEGFC/NRP2 autocrine loops. We previously described primary ccRCC cells obtained from surgically operated tumor specimens and normal epithelial cells from the same donor [15]. Tumor cells presented a wide range of sensitivity to NRPa-308 (from 40 to $0 \%$ 

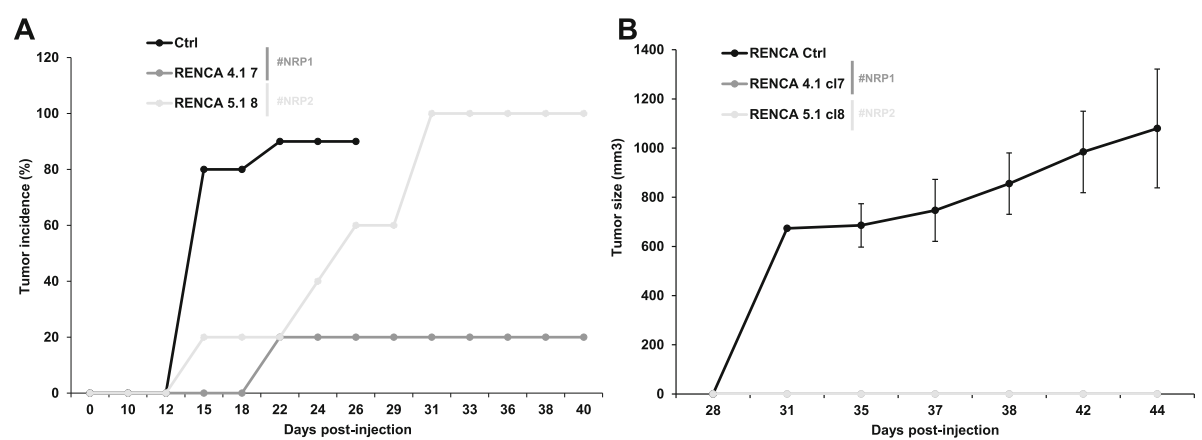

Fig. 2 NRPs KO inhibits experimental RCC growth in immunocompetent and immunodeficient mice. a Experimental tumors in nude mice were obtained after injection of $3 \times 10^{5}$ control (Ctrl, 10 mice) or NRPs KO RENCA cells ( 5 mice for each condition). One NRP1 KO clone (4.1 7) and one NRP2 KO clone (5.1 8) were injected. Tumor incidence (percentage of mice with tumors) at the indicated times is presented. $\mathbf{b}$ Experimental tumors in immuno-competent Balb-C mice ( 5 mice per condition) were obtained after subcutaneous injection of $3 \times 10^{5}$ control (Ctrl) or the above-mentioned NRPS KO RENCA cells. The tumor volume is represented for the indicated time

inhibition) as compared to normal primary kidney cells and to 786-O cells (Fig. 4c). TFE3 cells do not express VEGFC. Consequently, the NRP2/VEGFC pathway is not active in these cells. $4 \mathrm{C}$ cells express VEGFC equivalently to 786-O cells but do not express NRP2 and 4D cells express NRP2 but not VEGFC. In all the primary cells, the levels of VEGFA are modest and below to those of 786-O cells, hence limiting the activity of the NRP1/VEGFA pathway. These results suggest that the expression of NRPs and of their ligands VEGFA/VEGFC should be determined before the utilization of NRPa-308 in the clinic. Indeed, a difference in NRP1/VEGFA and/ or NRP2/VEGFC expression seem(s) to influence NRPa308 efficacy (Fig. 4d).

\section{NRPa-308-dependent inhibition of cell metabolic activity relied mainly on NRP2 in 786-O cells}

NRP1 and NRP2 KO cells constitute ideal tools to test the specificity and the NRPs- dependent effect on cell metabolic activity of NRPa-308. NRPa-308 was designed to inhibit VEGFA binding on NRP1. VEGFA and VEGF $C$ can interact with NRP1 and NRP2 and, as described above, VEGFA/NRP1 and VEGFC/NRP2 stimulate autocrine loops in ccRCC cells. Hence, we determined the

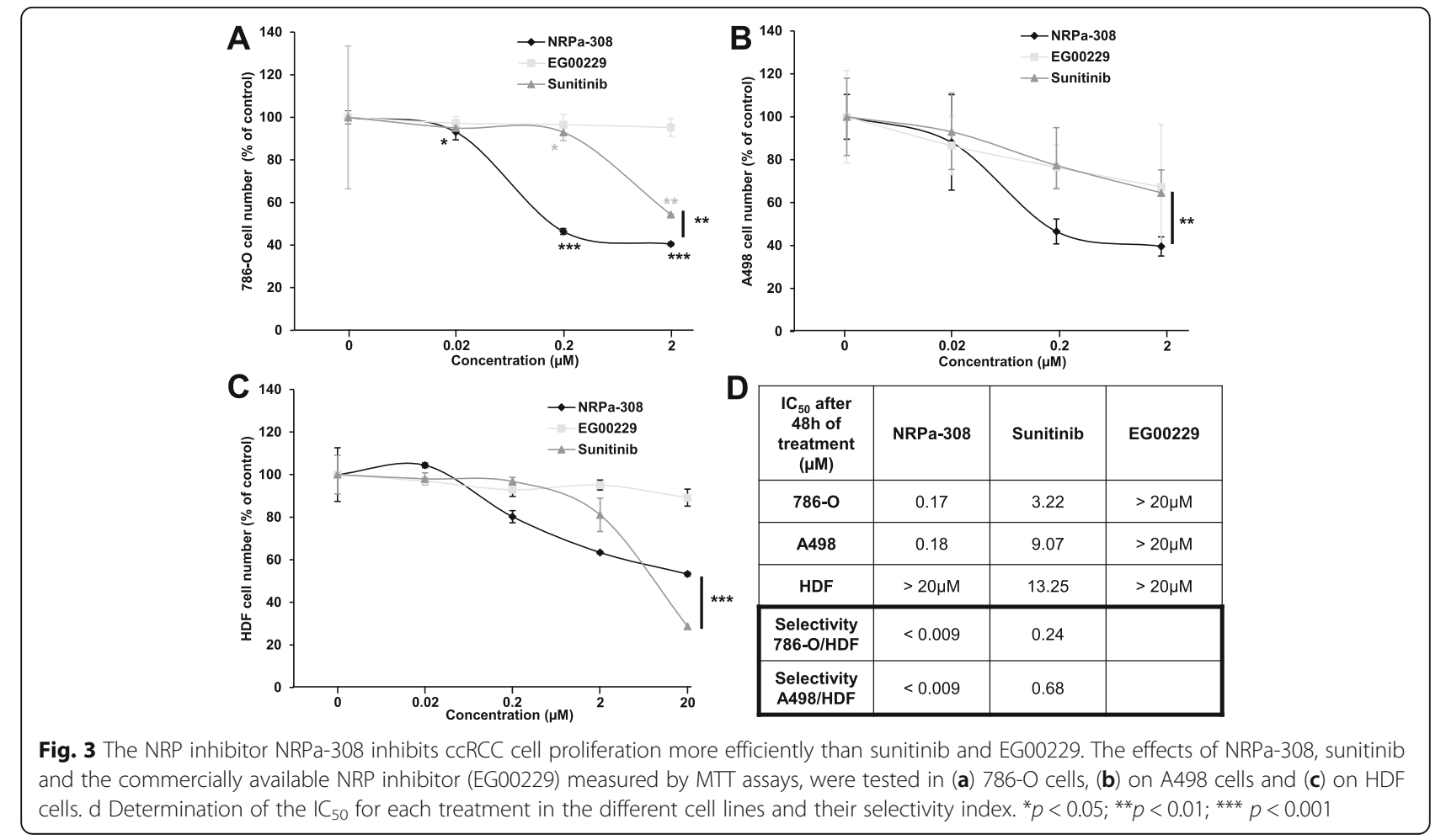



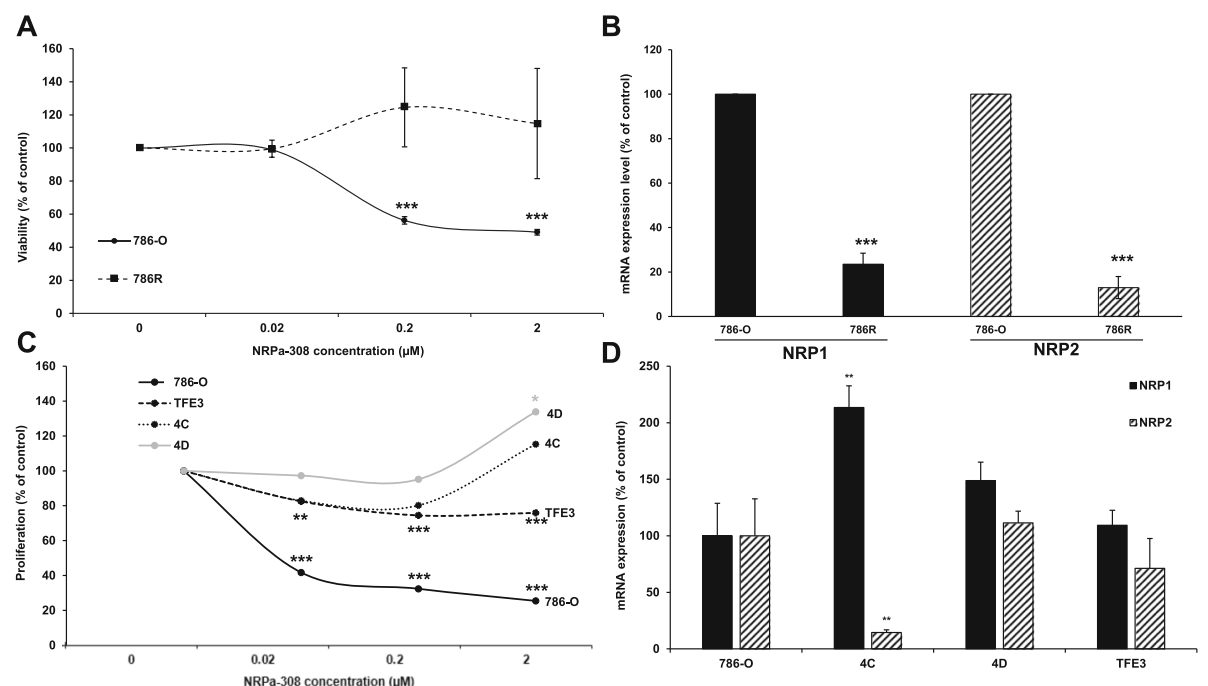

Fig. 4 NRPa-308 exerts a wide range of anti-proliferative effect on primary ccRCC cells. a The effects of NRPa-308 on cell viability were tested on 786-O (786-O) and 786-O cells resistant to sunitinib (786R). b The relative expression of NRP1 and NRP2 mRNA in 786-O and 786R cells was evaluated in a RNA seq analysis and confirmed by RT qPCR. $\mathbf{c}$ The sensitivity of NRPa-308 was tested on already described primary cells [15] by MTT assays. d The relative mRNA levels were evaluated in $786-0$ cells that served as reference values (100\%) and in the different primary cells

specific anti-metabolic activity of NRPa-308 in NRPs' $\mathrm{KO}$ clones. After $48 \mathrm{~h}$ of treatment, the $\mathrm{IC}_{50}$ of NRPa308 was increased in each clone. This result strongly suggests that NRPa-308 exerts its anti-metabolic activity via NRP1 and NRP2. However, the increase in $\mathrm{IC}_{50}$ was higher for the NRP2 KO clones, which suggest that NRPa-308 exerts its effects mainly via NRP2 (Fig. 5a). Clonogenic tests were also performed to further confirm the NRP2-dependency. No clone at all developed if NRPa-308 was removed in the culture medium of control or NRP1-KO cells. However, some clones emerged following treatment arrest of NRP2 KO clones which strongly suggests that NRPa-308 exerts its effects mainly via NRP2 (Fig. 5b). Affinity tests have been carried out to determine the efficacy of NRPa-308 to inhibit the VEGFA binding on NRP1 or NRP2 and the VEGFC binding on NRP2. The maximal inhibition reached approximately $40 \%$. NRPa-308 inhibited VEGFA/NRP1 and VEGFA/NRP2 binding in a dose-dependent manner but surprisingly, inhibited VEGFC/NRP2 binding in a reverse dose-dependent manner. Hence, low doses of NRPa-308 are sufficient to prevent VEGFC binding to NRP2 which also suggests a stronger affinity for NRP2 as compared to NRP1 (Fig. 5c).

\section{NRPa-308 binding mode is different between NRP1 and NRP2}

To understand the mechanisms linked to the inhibition of VEGFA/VEGFC binding to NRP1 and NRP2, we conducted a docking study. The NRPa-308 predicted binding mode completely differs between NRP1 and NRP2 (Fig. 6a). The orientation of NRPa-308 into NRP1 binding site is flipped relatively to those obtained into the NRP2 binding site. In both cases, NRPa-308 is stabilized in the binding site through hydrogen bonds, $\pi$-stacking and hydrophobic interactions (Fig. 6b), but most of the interacting residues are distinct. Few residues involved in these interactions are conserved in NRP1 and NRP2 (W301/ 304, S346/349, E348/351, Y353/356 according to the NRP1/NRP2 numeration) but they establish interactions with different parts of NRPa-308. Comparison of the NRP1 and NRP2 structures revealed that the residues forming each binding site differ and consequently the NRP2 binding site is larger and more open than the NRP1 binding site. This result explains the docking study obtained with NRPa-308 but also the difference of affinity experimentally obtained (Fig. 5c).

\section{NRPa-308 inhibited 786-O cell migration and invasion}

As described above, NRPs down-regulation and KO resulted in the inhibition of cell migration. Therefore, the ability of NRPa-308 to inhibit this parameter of tumor cell aggressiveness was tested. NRPa-308 reduced 786-O cell migration more efficiently than sunitinib at a very low concentration ( $0.02 \mu \mathrm{M}$ compared to $2 \mu \mathrm{M}$ for sunitinib, Fig. 7a-b). NRPa308 prevented also the ability of 786-O cells to invade a collagen matrix in a transwell system (Fig. 7c). This result suggests an anti-metastatic activity of NRPa-308.

High NRPa-308 concentration stimulated the production of NRPs' ligands and of pro-angiogenic/pro-inflammatory cytokines

We observed that NRPs KO resulted in increased production of their ligands (Fig. 1). Although these ligands 


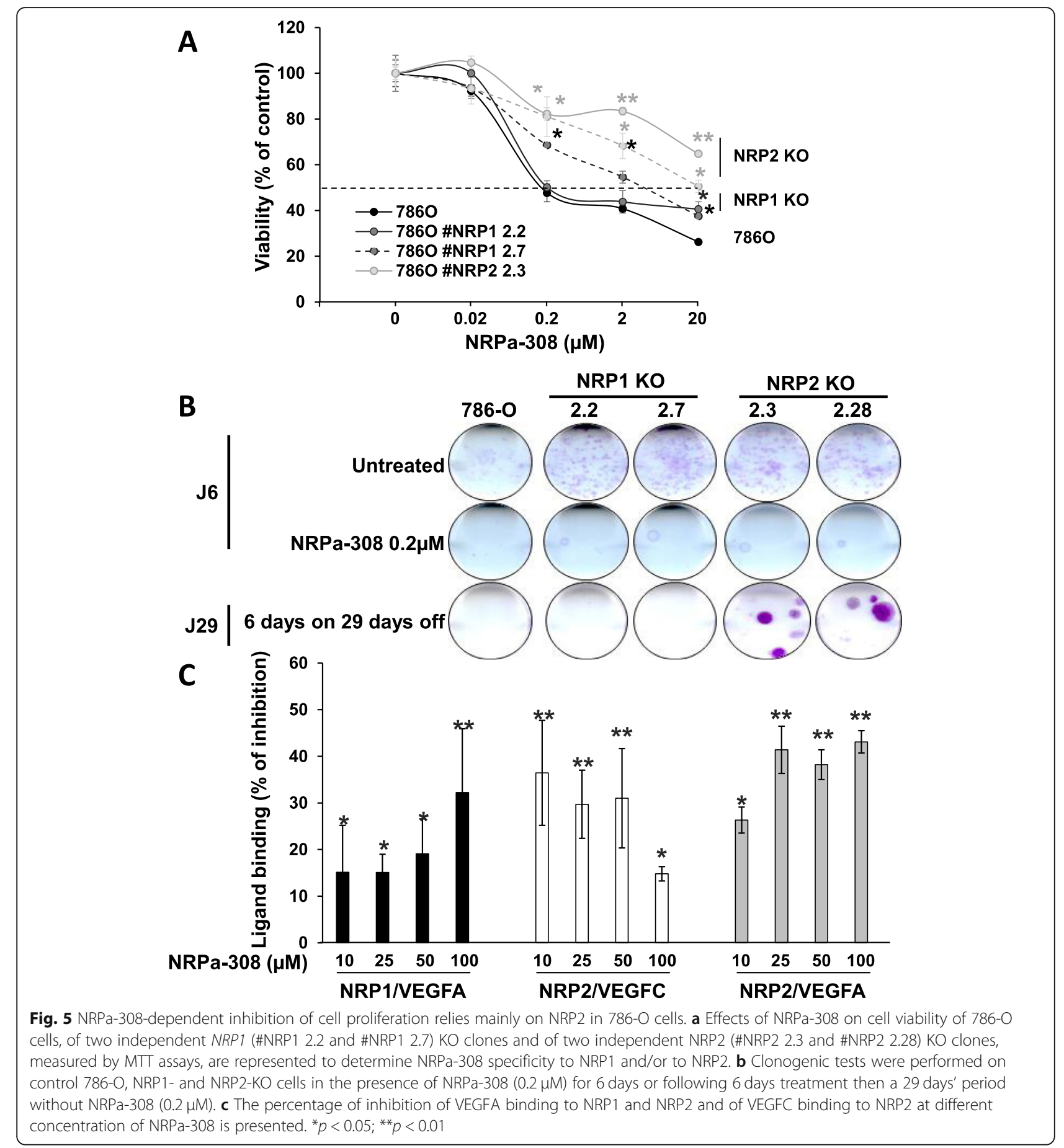

cannot influence tumor cells KO for NRPs, their paracrine effects can be highly detrimental by stimulating angio/lymphangiogenesis and by inducing immunotolerance. Hence, we evaluated the minimal NRPa-308 concentration, which inhibits cell metabolic activity without influencing their secretome. $0.2 \mu \mathrm{M}$ of NRPa-308 maximally decreased the percentage of metabolically active cells (Fig. 3). Increasing further the concentration did not result in a better efficacy of the drug. Hence, we analyzed the expression of VEGFA and VEGFC following NRPa-308 treatment. We also determined the expression of the ELR + CXCL cytokines CXCL5 and CXCL8 since they are involved in resistance to bevacizumab and sunitinib in ccRCC as we previously described [16, 28]. Sunitinib, at these low concentrations (below the $\mathrm{IC}_{50}$ [27]), had no influence on VEGFA and VEGFC expression but 

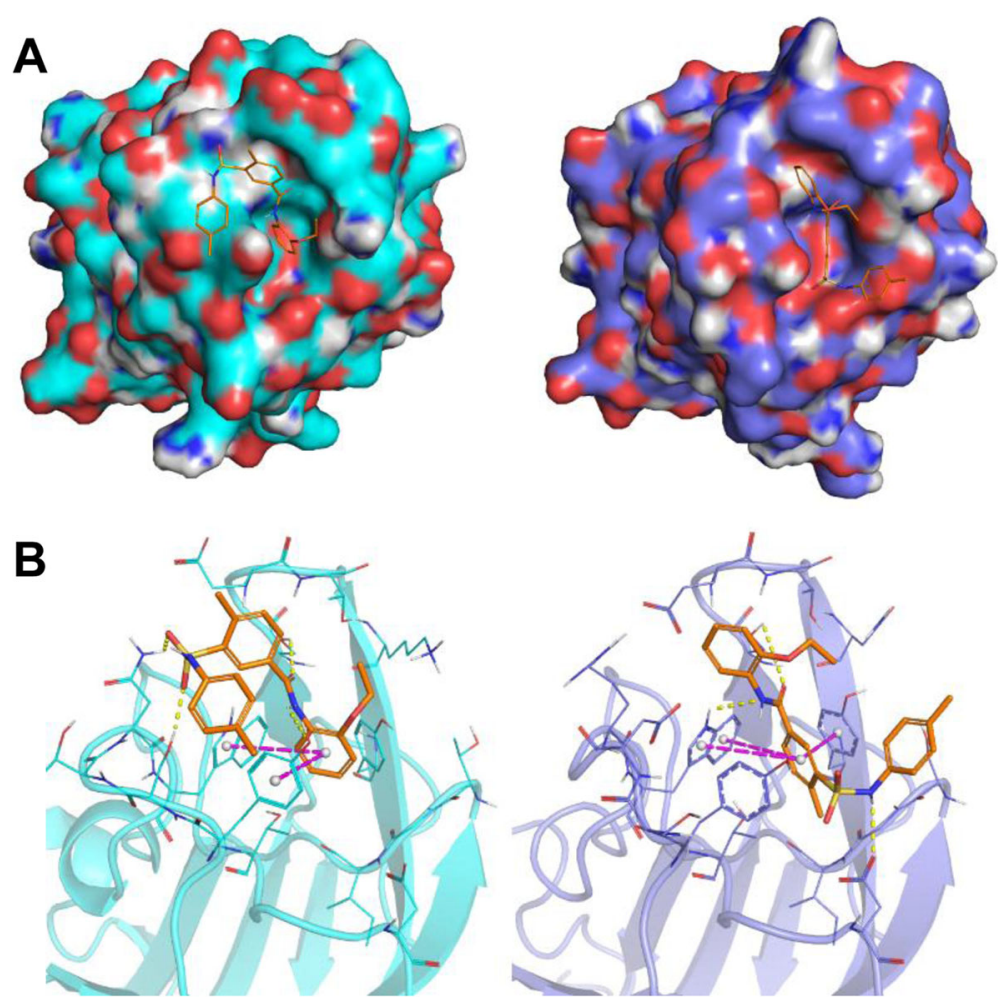

Fig. 6 NRPa-308 binding mode is different between NRP1 and NRP2. NRPa-308 (colored in orange) predicted binding mode into the NRP1 (a and $\mathbf{b}$ in cyan, left panels) and NRP2 ( $\mathbf{a}$ and $\mathbf{b}$ in blue, right panels) binding sites. Hydrogen bonds are depicted as yellow dashed lines and $\pi$-stacking are depicted as magenta dashed lines

increased CXCL5 and CXCL8 expression as previously shown (Fig. 8a-d) [28]. NRPa-308 increased the expression of these different factors at the highest concentration $(2 \mu \mathrm{M})$. The lowest concentration $(0.2 \mu \mathrm{M})$, only stimulated the expression of VEGFC. These results strongly suggest that the best ratio (beneficial/detrimental effects) can be obtained at low doses of NRPa-308 in the context of ccRCC.

\section{NRPa-308 decreased experimental ccRCC growth in a reverse dose-dependent manner}

In previous studies [8], NRPa-308 inhibited the growth of experimental breast cancers at an optimal dose of $50 \mathrm{mg} /$ $\mathrm{kg}$. A pilot experiment on experimental ccRCC generated in nude mice with 786-O cells was unsuccessful (no inhibition of tumor growth). The results presented in Fig. 8 suggested detrimental paracrine effects induced by high concentrations of NRPa-308. Therefore, we tested the effects of increasing concentrations of the drug $(5 \mu \mathrm{g} / \mathrm{kg}$, $500 \mu \mathrm{g} / \mathrm{kg}$ and $50 \mathrm{mg} / \mathrm{kg}$ ) on the growth of experimental ccRCC in immunodeficient (xenograft of human 786-O cells) and immunocompetent (graft of syngenic mouse RENCA cells) mice. Considering a full distribution in the blood and a $1.5 \mathrm{ml}$ of blood in a mouse of $25 \mathrm{~g}$, the respective blood concentrations of the drug administered at $5 \mu \mathrm{g} / \mathrm{kg}, 500 \mu \mathrm{g} / \mathrm{kg}$ and $50 \mathrm{mg} / \mathrm{kg}$ should be around 0.2 , 20 and $2000 \mu \mathrm{mol} / \mathrm{L}$. Of course, these blood concentrations correspond to a rough estimation which does not consider the biological distribution in the organs especially metabolism of the drug in the liver. The lowest concentration is in the range of concentrations inhibiting cell metabolic activity, migration and invasion and impacting to a low extent the production of pro-angio/lymphangiogenic and pro-inflammatory cytokines. The highest NRPa-308 dose did not affect the growth of experimental ccRCC. However, tumor growth was inhibited significantly by the lower amounts, especially the lowest concentration of $5 \mu \mathrm{g} / \mathrm{kg}$ in both mouse models (Fig. 9a-b). Immunostaining were carried out on tumors generated in immunodeficient mice. The number of blood vessels (CD31 labeling) and of pericytes/cancer associated fibroblast (CAF, $\alpha$ SMA labeling) per $\mathrm{cm}^{2}$ was high in the control group and increased in a dose-dependent manner (Fig. 9c-d). However, the number of arterioles (CD31/ $\alpha$ SMA co-labeling) decreased in a dose-dependent manner (Fig. 9e-f). Lymphatic vessels in these tumors were almost undetectable and NRPa-308 did not modify their number. These experiments showed that NRPa-308 represents an interesting 


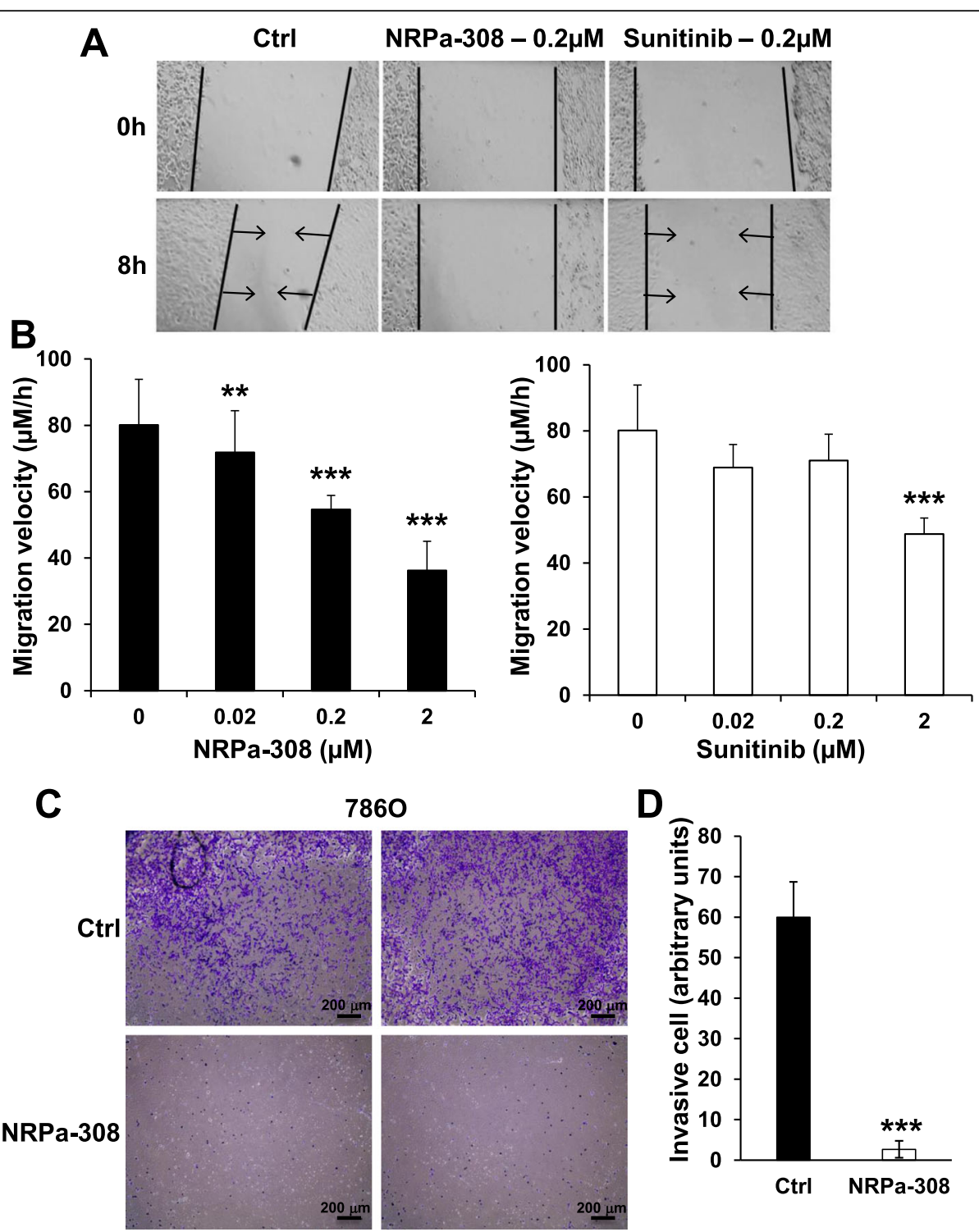

Fig. 7 NRPa-308 inhibits 786-O cell migration velocity more efficiently than sunitinib. a Photographs of scratch assay on cell monolayers in different experimental conditions; untreated, treated by NRPa-308 and by sunitinib. $\mathbf{b}$ The effects of NRPa-308 and sunitinib on 786-O cell migration at different concentrations by quantifying the above-mentioned experiments. c NRPa-308 $(2 \mu \mathrm{M})$ inhibited the invasion of collagen-coated Boyden chambers (Control (Ctrl) and NRPa-308-treated duplicates are shown). d Quantification of the results shown in (c). ${ }^{* *} p<0.01 ;{ }^{* * *} p<0.001$

therapeutic strategy for ccRCC at a low concentration which is a good compromise associating efficacy and low toxicity (no modification of mouse weight at low doses, Fig. S5).

\section{Efficient NRPa-308 dose decreased the expression of pro- tumoral factors}

To understand the better efficacy of low doses of NRPa308 , we evaluated the expression of genes involved in tumor aggressiveness especially those regulating proliferation, angio/lymphangiogenesis, epithelial/mesenchymal transition (EMT) and immune tolerance. The modifications to their mRNAs, analyzed by qPCR, were compiled in Fig. 10. Genes associated with lymphangiogenesis were the most downregulated by the lowest dose of the drug including human NRP2, Prox1 and VEGFC and murine Prox1 and VEGFC in the immunodeficient model (Fig. 10a) and NRP2, Prox1 and VEGFC in the immunocompetent model (Fig. 10b). Only murine Prox1 and VEGFC were downregulated by the intermediate dose in the immunodeficient model (Fig. 10a). Human NRP2, Prox1, and VEGFC and murine NRP2 were upregulated in the presence of the highest dose in the immunodeficient model (Fig. 10a). In the immunodeficient model, proangiogenic genes including human NRP1, VEGFA and murine NRP1, VEGFA, VEGFR1 and VEGF 

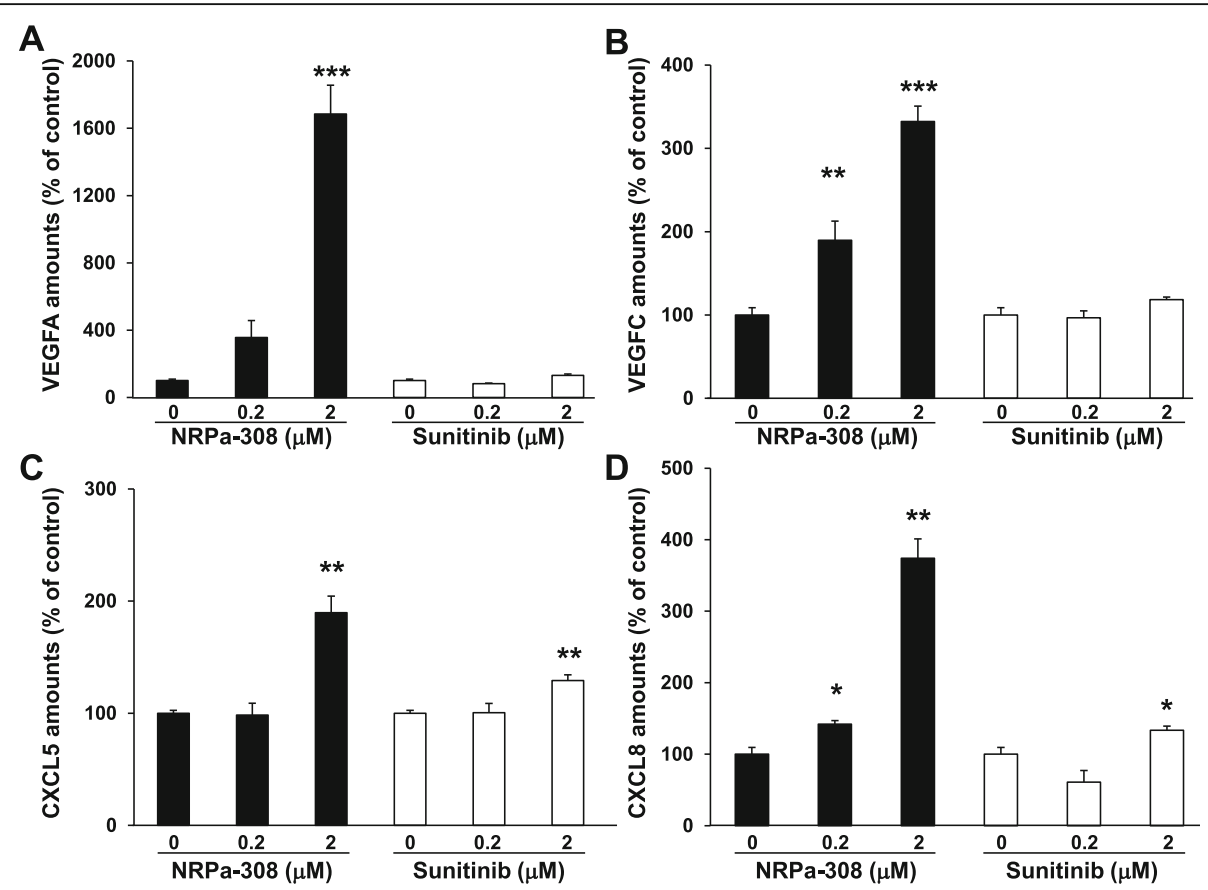

Fig. 8 High NRPa-308 concentration stimulates the production of NRPs ligands and of pro-angiogenic/pro-inflammatory cytokines. The effects of NRPa-308 and sunitinib on the production of different cytokines were evaluated by ELISA; a VEGFA, b VEGFC, production (c) CXCL8, d CXCL5. ${ }^{*} p<0.05 ;{ }^{* *} p<0.01 ;{ }^{* * *} p<0.001$

R2 were downregulated by the highest dose (Fig. 10a). Some of them were also downregulated by the lowest or intermediate dose including human VEGFA and VEGF R1 and murine NRP1, VEGFR1 and VEGFR2 (Fig. 10a). Human NRP1 and VEGFR1, and murine VEGFA and VEGFR2 were upregulated by using the lowest or the highest dose (Fig. 10a). In the immunocompetent model, the proangiogenic genes NRP1 and VEGFR1 were downregulated for the two doses (Fig. 10b). The murine gene involved in immunotolerance, PDL1 was downregulated by the lowest and intermediate dose and was unchanged for the highest dose in immunodeficient mice (Fig. 10a). It was downregulated by the two doses in immunocompetent mice. In the immunodeficient model, genes involved in EMT including human MET and HGF and murine MET and HGF were downregulated by the lowest or intermediate dose (Fig. 10a). Only murine MET was downregulated by the highest dose and human MET and HGF and murine HGF were upregulated by the intermediate or highest dose (Fig. 10a). In the immunocompetent model MET and HGF were downregulated by the two doses (Fig. 10b). In the immunodeficient model, mCD69, a marker of the lymphocytes' activation, is upregulated, which is synonymous of an activation of the immune response (Fig. 10a). The M2 macrophages marker mARG1 was decreased, which reflects a beneficial polarization of macrophages (Fig. 10a). According to these differences, we attempted to establish a score of good or bad prognosis depending on the up or downregulation of genes involved in tumor aggressiveness. This score will serve to understand why we did not observe a dose dependent effect of NRPa308. We gave a score of 2 when a gene of poor prognosis decreased and a score of -2 when it increased and vice versa for a gene of good prognosis. The global score for the lowest concentration was respectively 18 and 12 in the immunodeficient (Fig. 10a) and immunocompetent models (Fig. 10b). It was of 20 for the intermediate dose in the immunodeficient model (Fig. 10a) and of 2 and 6 respectively for the highest dose in the immunodeficient (Fig. 10a) and the immunocompetent models (Fig. 10b). This evaluation, in addition to the reduction of tumor growth consistently favored the notion that a low dose of NRPa-308 had the best therapeutic efficacy that was not counterbalanced by the expression of genes related to tumor aggressiveness.

\section{The NRP2 associated pathway is more relevant for the aggressiveness of mccRCC}

We were puzzled by confronting our previous results on breast cancers with those enclosed in this manuscript; high dose of NRPa-308 was the most efficient on models of breast cancers whereas it has no effect in models of ccRCC [8]. Considering the striking therapeutic value of targeting NRPs for both models of cancers, we first analyzed the relative expression of NRPs and their ligands 


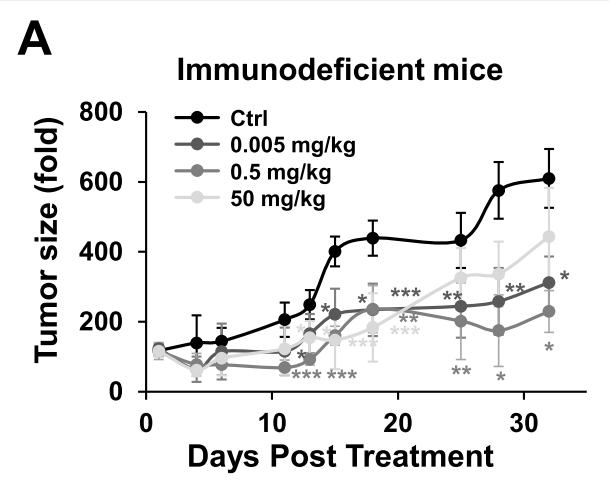

B
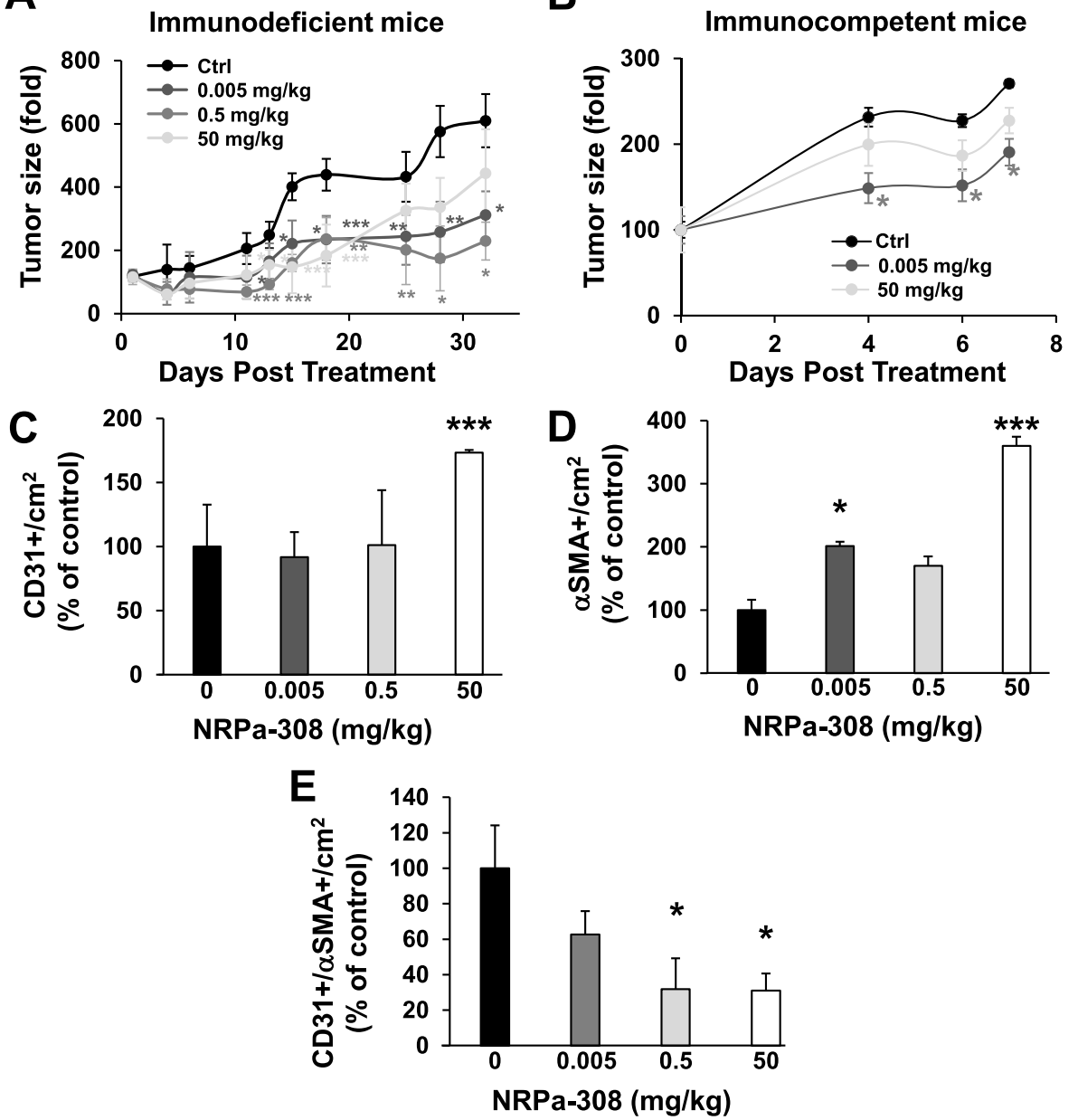

F

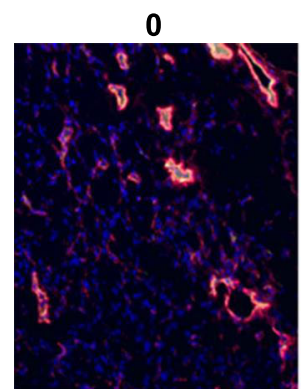

DAPI

\section{NRPa-308 (mg/kg)}
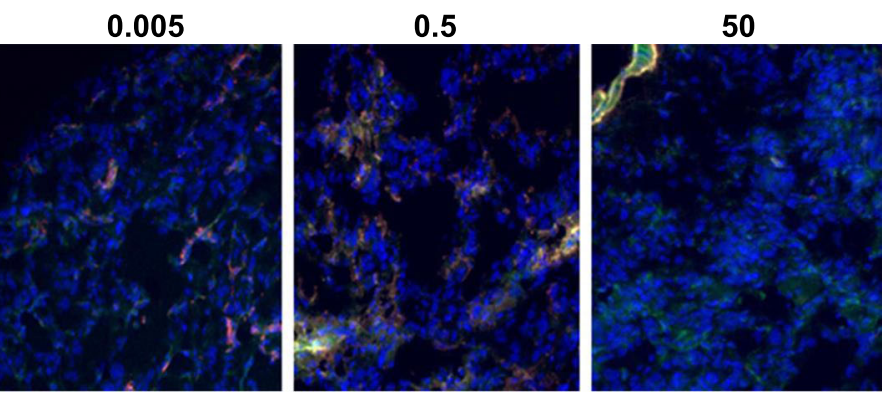

Fig. 9 NRPa-308 decreases experimental ccRCC growth in a reverse dose-dependent manner. a Experimental tumors in nude mice (5 mice per condition) were obtained after injection of $3 \times 10^{6} 786-0$ cells. Three concentrations of NRPa-308 (5 $\mu \mathrm{g} / \mathrm{kg}, 500 \mu \mathrm{g} / \mathrm{kg}$ and $\left.50 \mathrm{mg} / \mathrm{kg}\right)$ diluted in carboxymethyl cellulose, were given trice a week by oral gavage. The control group (Ctrl) received carboxymethyl cellulose. Tumor volume represented as a fold increase from the beginning of the treatment is presented. $\mathbf{b}$ Experimental tumors in immunocompetent mice (Balb-C, 5 mice per condition) were obtained after injection of $3 \times$ $10^{5}$ RENCA cells. Treatment (NRPa-308) was given trice a week by oral gavage. Two concentrations of NRPa-308- (5 $\mu \mathrm{g} / \mathrm{kg}$ and $\left.50 \mathrm{mg} / \mathrm{kg}\right)$ diluted in

carboxymethyl cellulose were administered. The control group (Ctrl) received carboxymethyl cellulose. Tumor volume represented as the fold increase from the beginning of the treatment is shown. The tumor vasculature in each experimental group was detected by immuno-staining for CD31 (endothelial cells, green) and a-SMA (pericytes, cancer associated fibroblasts), red). Tumor sections were counterstained with 40,6-diamidino-2-phenylindole (DAPI) (nucleus, blue). c Quantification of the blood vessels (CD31 labeling). d Quantification of pericytes and cancer associated fibroblasts (a-SMA). e Quantification of blood vessels covered with pericytes (yellow labeling). $\mathbf{f}$ Representative images of the dose-dependent decrease in blood vessels covered with pericytes. ${ }^{*} p<0.05$; ${ }^{* *} p<0.01$; *** $p<0.001$ 
A

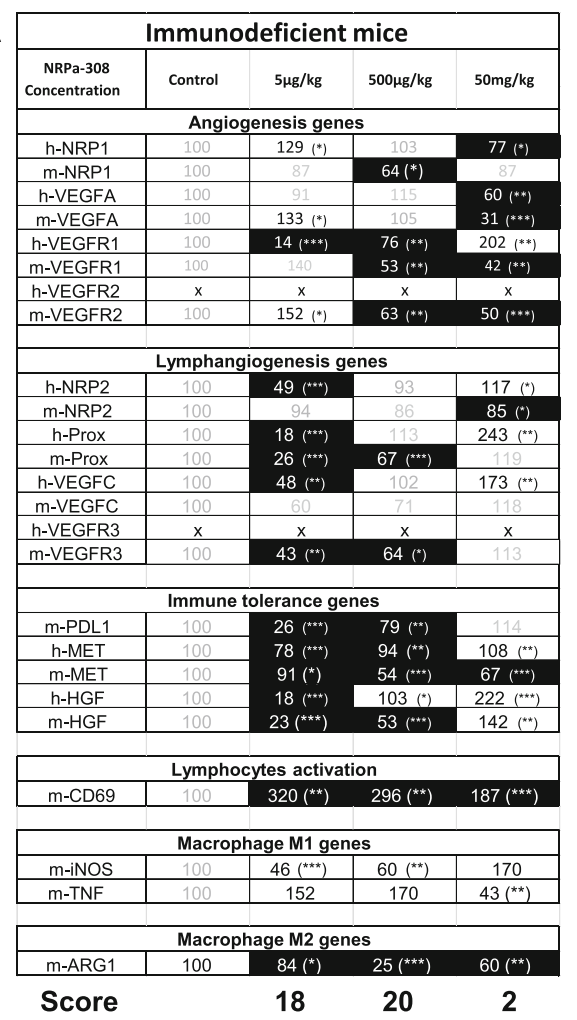

B

\begin{tabular}{|c|c|c|c|}
\hline \multicolumn{4}{|c|}{ Immunocompetent mice } \\
\hline $\begin{array}{c}\text { NRPa-308 } \\
\text { Concentration }\end{array}$ & Control & $5 \mu \mathrm{g} / \mathrm{kg}$ & $50 \mathrm{mg} / \mathrm{kg}$ \\
\hline \multicolumn{4}{|c|}{ Angiogenesis genes } \\
\hline m-NRP1 & 100 & $9^{(* * *)}$ & $52(* * *)$ \\
\hline m-VEGFR1 & 100 & $1(* * *)$ & $1\left(^{* * *}\right)$ \\
\hline \multicolumn{4}{|c|}{ Lymphangiogenesis genes } \\
\hline m-NRP2 & 100 & $\left.288^{(\star \star}\right)$ & 103 \\
\hline m-Prox & 100 & $26\left(^{(* \star}\right)$ & 150 \\
\hline m-VEGFC & 100 & $22(* * *)$ & 139 \\
\hline m-VEGFR3 & 100 & $173\left(^{*}\right)$ & $\left.626{ }^{(* *}\right)$ \\
\hline \multicolumn{4}{|c|}{ Immune tolerance genes } \\
\hline m-PDL1 & 100 & $26\left(^{(* *)}\right.$ & $51(* *)$ \\
\hline CTLA4 & $x$ & $x$ & $x$ \\
\hline m-MET & 100 & $\left.0.2^{(* \star}\right)$ & $4\left(^{(\star \star}\right)$ \\
\hline m-HGF & 100 & $3\left(^{(\star \star}\right)$ & 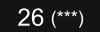 \\
\hline \multicolumn{4}{|c|}{ Macrophage M1 genes } \\
\hline m-iNOS & 100 & $73\left({ }^{(\star \star}\right)$ & $16\left(^{\star \star \star}\right)$ \\
\hline Score & & 12 & 6 \\
\hline
\end{tabular}

Fig. 10 Efficient NRPa-308 dose decreases the expression of pro-tumoral factors. Detection by QPCR of pro-tumoral genes in tumors generated in immunodeficient and immunocompetent mice with wild-type and with NRPs knock-out cells. ${ }^{*} p<0.05 ;{ }^{* *} p<0.01 ;{ }^{* * *} p<0.001$

VEGFA and VEGFC on a panel of cell lines available in the TCGA data base. In most of the cell lines representative of aggressive ccRCC and breast cancers, VEGFA and NRP1 are expressed at high levels especially in the cell lines used in our respective experimental tumor growth (786-O and MDAMB231) (Fig. 11a-c). VEGFC and NRP2 are expressed by all the ccRCC cell lines. However, VEGFC levels are very low in three out of five breast cancer cell lines and NRP2 levels are very low in all the breast cell lines including MDAMB231 (Fig. 11bd). These very low levels in MDAMB231 and the more specific effects of NRPa-308 on NRP2, partly explained the results obtained on experimental tumor growth. Our next step was to deep insight into the prognostic role of NRP1 and NRP2 and their known partners VEGFA, VEGFR1, VEGFR2, Semaphorin 3A (Sema3A) and plexin A1 (PLXNA1) (all NRP1 partners) and VEGFC, VEGF R3, Semaphorin 3F (Sema3F), plexin A2 (PLXNA1) and Prospero homeobox protein 1 (Prox1), a master transcription factor of lymphangiogenesis (all NRP2 partners). For that purpose, we correlated the expression of these different partners to disease free survival (DFS, non-metastatic patients $\mathrm{M} 0$ ), progression free survival (PFS, metastatic patients M1) and overall survival (OS) in patients with ccRCC and in patients with the most severe triple negative breast cancers (TNBC). For each gene, we defined the best cut off that determines a survival difference. Four hundred twenty-five samples were from M0 and 103 from M1 ccRCC patients. One hundred fifteen samples were from TNBC patients.

For M0 ccRCC patients, expression of VEGFR2, NRP2, VEGFC, VEGFR3, PLXA2 above their respective best cut off was of good prognosis for DFS (trend ( $T$, p between 0.08 and 0.06) for NRP2, VEGFC) and significant (S) for VEGFR2, VEGFR3, PLXA2). Expression above the best cut off for Sema3A and Prox1 was of poor prognosis for DFS. For M0 patients OS, NRP1, VEGFR1, VEGFR2, NRP2, VEGFC, VEGFR3, Sema3F and PLXA2 (T for NRP1, VEGFC, VEGFR3 $\mathrm{S}$ for VEGFR1, VEGFR2, NRP2 and Sema3F). For M1 ccRCC patients, only Sema3F (T) was correlated to a longer PFS whereas eight parameters were correlated to a worse prognosis (NRP1 (S), VEGFR2 (S), Sema3A (S), NRP2 (S), VEGFC (S), VEGFR (S)3, PLXA2 (T) and Prox1 (S)). NRP1 (S), VEGFR1 (S), VEGFR2 (S), Sema3F (S) and PLXA2 (S) were correlated with a longer OS while Sema3A (S), PLXA1 (S), NRP2 (S), VEGFC (S) and Prox1 (S) were correlated to a shorter one.

For TNBC, VEGFA was curiously associated with a longer PFS (T) but NRP1 (S), VEGFR1 (S), PLXNA1 (S), NRP2 (T), VEGFC (T), Sema3F (S) and PLXNA2 (T) were correlated with a shorter one. NRP1 (S), VEGFR1 

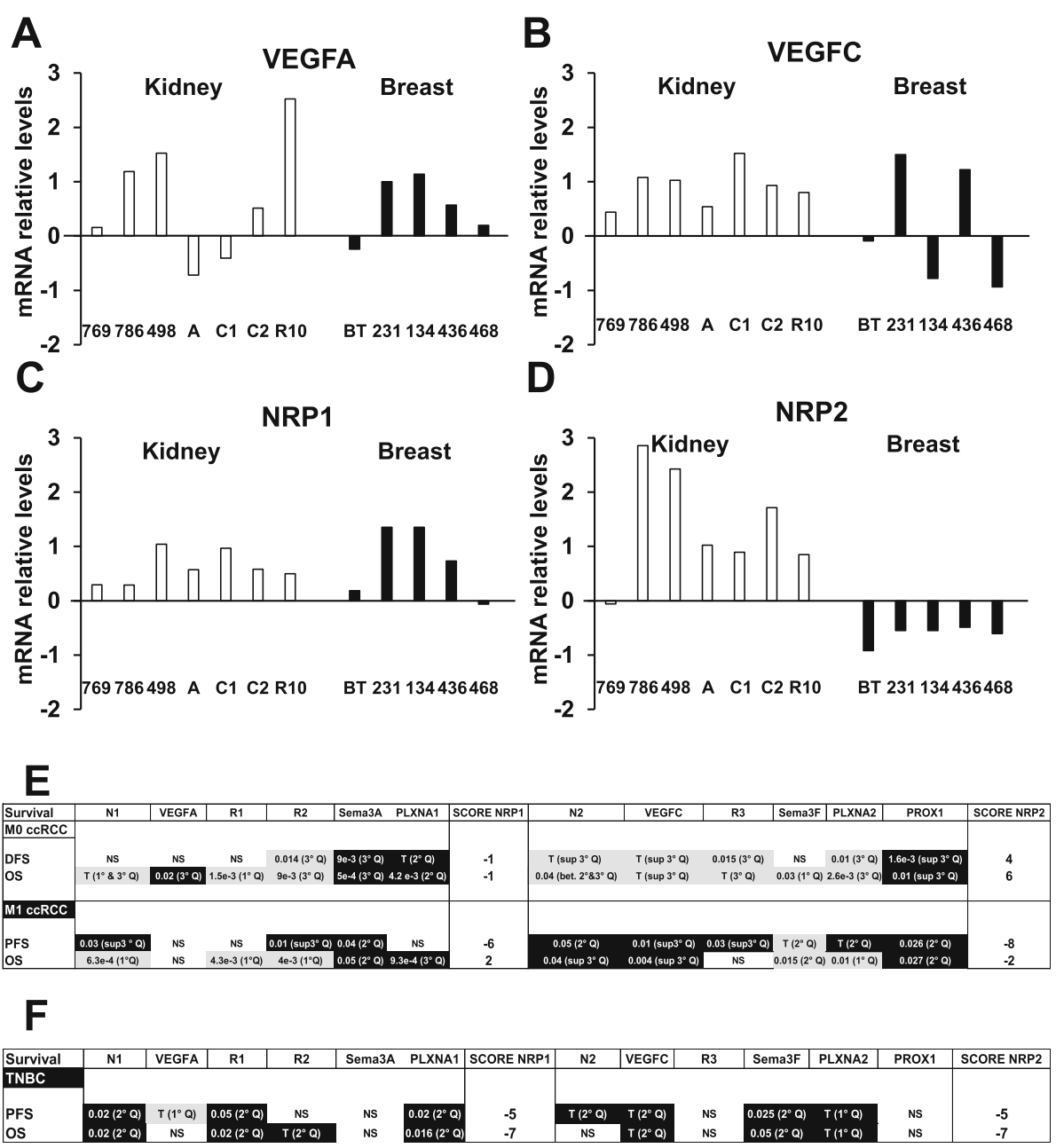

Fig. 11 The NRP2 associated pathway is more determinant for the aggressiveness of mccRCC but not for triple negative breast cancers. Analysis of cbioportal database highlighted the relative levels of VEGFA (a), VEGFC (b), NRP1 (c) and NRP2 (d) mRNA in a panel of RCC (769 (769P), 786-O (786), ACHN (A), Caki1 (C1), Caki2 (C2), RCC10 (R10)) and TNBC (BT474 (BT), MDAMB231 (231), MDAMB134 (134), MDAMB436 (436), MDAMB468 (468)). Correlation between genes of the NRP1 and NRP2 pathways and survival (DFS/PFS/OS) in M0 and M1 RCC patients (e) and TNBC (f) patients. The tested genes of the NRP1 pathway were the following: NRP1 (N1), VEGFA, VEGFR1 (R1), VEGFR2 (R2), Semaphorin 3A (Sema3A), Plexin A1 (PLXNA1). The tested genes of the NRP2 pathway were the following: NRP2 (N1), VEGFC, VEGFR3 (R3), Semaphorin $3 F$ (Sema3F), Plexin A2 (PLXNA1) and PROX1. The $p$-values of genes associated with shorter DFS/PFS/OS appear white on a black background; the $p$-values of genes associated with a longer DFS/PFS/OS appear black on a gray background. Significant p-values are given; a trend to significance is indicated by a "T". Specific cut-off are indicated (First, second or third quartile $\left(1^{\circ}, 2^{\circ}, 3^{\circ} \mathrm{Q}\right)$. A score was established as follows: a positive point was given for a gene with a trend to good prognosis; two positive points for a gene associated with good prognosis and with a significant p-value; a negative point was given for a gene with a trend to poor prognosis; two negative points were given for a gene associated with poor prognosis and with a significant p-value. Positive scores were obtained for DFS and OS of MO RCC patients and the NRP2 pathway (respectively 4 and 6) and for the OS of M1 RCC patients and the NRP1 pathway (2). Negative scores were obtained for obtained for the DFS and OS of M0 and PFS of M1 RCC patients and the NRP1 pathway (respectively $(-1),(-1)(-6)$, for the PFS and OS of M1 RCC patients and the NRP2 pathway. Negative score were obtained for the NRP1 and NRP2 pathways for PFS and OS

(S), VEGFR2 (T), PLXNA1 (S), VEGFC (T), Sema3F (S) and PLXNA2 $(\mathrm{T})$ were correlated with a shorter OS. NRP2 was not correlated to survival in that case.

We defined a score by attributing a relative weight of -2 for a gene associated with a significant poor prognosis and a relative weight of -1 for a trend. Inversely, a relative weight of 2 was given for a gene associated with a significant good prognosis and 1 for a trend. NRP1 and NRP2 pathways were considered separately. For the NRP1 pathway, a-1 score was obtained for the DFS and OS of M0 ccRCC patients, -6 and 2 scores for PFS and OS of M1 ccRCC patients, and - 5 and -7 scores for the PFS and OS of TNBC patients. For the NRP2 pathway, positive score of 4 and 6 were obtained for the DFS and OS of ccRCC patients, -8 and -2 scores for M1 ccRCC patients and -5 and -7 scores for the PFS and OS of TNBC patients. 
These results showed that NRP1 and NRP2 signaling pathways, in general, strongly correlate with shorter survival for the most aggressive cancers, M1 ccRCC and TNBC. However, NRP2 is correlated with shorter DFS and OS in ccRCC while NRP1 is more involved in TNBC patients' survival. These results suggest that NRP1 targeting is more adapted for TNBC while NRP2 targeting is more adapted for ccRCC.

\section{Discussion}

NRPs, through their direct effect on tumor cells (stimulation of metabolic activity and migration/invasion) and on cells of the microenvironment (angio/lymphangiogenesis and immune tolerance) are key signaling molecules stimulating ccRCC growth and metastasis. However, the multi partnerships of NRPs render difficult the determination of the relative importance of each pathway. Moreover, we discovered that NRP1 and NRP2 signaling cross-talked to establish a steady state depending on the production of VEGFA and VEGFC. This phenomenon was strikingly observed in NRP1 KO clones in which compensatory expression of NRP2 was insufficient to inhibit cell migration (Fig. 1f). We also showed that inflammatory cytokines compensate for the inhibition of NRPs' pathways. These compensatory mechanisms are key for an optimized targeting of NRPs in the context of ccRCC treatment. Our results had to be compared to those of Cao $\mathrm{Y}$ et al who showed that inhibition of experimental tumor growth generated with cells downregulated for NRPs only relies on microenvironment shaping $[10,29]$. By generating the same models, we showed discrepant results at longer time points as compared to those of Cao $\mathrm{Y}$ et al. These discrepancies depend on the stimulation of alternative autocrine pathways mediated by a modified secretome. However, modifications of these secretomes also depend on a partial or complete inhibition by $\mathrm{KO}$ of the NRPs' signaling. These compensatory mechanisms are particularly striking if NRPs' signaling inhibition enters in a therapeutic strategy. We are aware that $\mathrm{KO}$ or pharmacological inhibition may induce different responses. However, it was the only way to generate compelling evidence for demonstrating the relevance of targeting both NRPs for an optimal therapeutic strategy. The puzzling results that we obtained in the present and in our previous study, highlight the relative importance of NRP1 or NRP2 signaling depending on the cancer type. NRPa-308, discovered by its ability to inhibit VEGFA binding to NRP1 [13], is a better NRP2 inhibitor. Analysis of the TCGA database revealed that NRP1 is a better therapeutic target for TNBC and NRP2 is a better one for ccRCC. This result suggests that NRP1 or NRP2 inhibitors are more relevant for a specific cancer. The importance of the double $\mathrm{KO}$ was questioned in 786$\mathrm{O}$ cells. Several attempts were unsuccessful suggesting that the double $\mathrm{KO}$ is lethal. Hence, an inhibitor of NRP1/
VEGFA or NRP2/VEGFC is relevant but must not induce compensatory signaling pathways as for conventional antiangiogenic drugs [30]. Therefore, an inhibitor of both NRP1 and NRP2 will be more efficient.

Anti-angiogenic drugs, immunotherapies or the combo are the current standard of care [31, 32]. Our results suggest that resistance to anti-angiogenics especially sunitinib, involved a down-regulation of NRPs. Therefore, NRPs' inhibitors do not seem relevant at relapse on sunitinib. However, NRPs' inhibitors represent an alternative following failure of immunotherapies used in the first line treatment of M1 patients [14]. Adjuvant treatment for M0 ccRCC patients is a debated issue. While some trials showed that an adjuvant treatment by anti-angiogenics is not relevant, another trial demonstrated its importance for advanced M0 patients [33, 34]. Our results showed that the NRP2 pathway is correlated with a good prognosis for M0 patients and NRP1 did not correlate with shorter survival rates. Our results emphasized the relevance of NRPs targeting in only M1 ccRCC patients and anti NRPs should not be used in an adjuvant setting.

\section{Conclusions}

Comparison of ccRCC and TNBC highlighted the relevance of the NRP1 and the NRP2 pathways. Predetermination of NRPs' expression is important to administrate NRP inhibitors. Although NRPa-308 represents an interesting hit if tumor cells express both NRPs and their ligands VEGFA/VEGFC, specific drugs targeting NRP1 should be more appropriate if only NRP1/VEGFA is present. This concept must be further addressed in depth to reach the "golden age" of the therapeutic arsenal for cCRCC [35].

\section{Supplementary Information}

The online version contains supplementary material available at https://doi. org/10.1186/s13046-021-01832-x.

Additional file 1: Table S1. List of oligonucleotides used in GPCR experiments. Table S2. Recapitulative table of the expression of NRP1 and NRP2 in KO and knock-down cells. Table S3. Recapitulative table of the expression of VEGFA and VEGFC in KO and knock-down cells. Table S4. NRP1 and NRP2 binding site descriptors computed with DogSite Scorer (Nb: number, HBA: Hydrogen Bond Acceptor, HBD: Hydrogen Bond Donor, AA: amino acids)

Additional file 2: Fig. S1. Study of down-regulation of NRPs by shRNA in 786-O cells. (A-B) Effects of the downregulation of NRP by shRNA on NRP1 and NRP2 mRNA expression measured by qPCR. (C) Effects on cell metabolic activity measured by MTT assays. (D) Down-regulation of NRPs decreased cell migration. Bevacizumab increased this effect for NRP1 down-regulation. (E) Down-regulation of NRPs had no effect on VEGFA and VEGFC production measured by ELISA. ${ }^{*} p<0.05 ;{ }^{* *} p<0.01 ;{ }^{* * *} p<$ 0.001 .

Additional file 3: Fig. S2. Effects of NRP1 or NRP2 gene invalidation in RENCA cells. (A) NRP1 and NRP2 protein levels were evaluated by flow cytometry in control (RENCA), in \#NRP1 4.1.7 and \#NRP2 5.1.8 clones. (B) Effects of NRPS KO on RENCA cell metabolic activity measured by MTT 
assays. (C) Effects of NRPs KO in RENCA cells on the VEGFA and VEGFC protein levels measured by ELISA. ${ }^{*} p<0.05$; ${ }^{* *} p<0.01$; ${ }^{* * *} p<0.001$.

Additional file 4: Fig. S3. NRPs KO in 786-O tumor cells inhibited experimental RCC growth in immunodeficient mice. (A) Experimental tumors in nude mice (5 mice per condition) were obtained after injection of $3 \times 10^{6}$ wildtype (Ctrl) or NRPs KO 786-O cells. One NRP1 (\#NRP1 2.7) clone and one NRP2 (\#NRP2 2.3) clone were injected. Tumor volume is presented. ${ }^{*} p<0.05 ;{ }^{* *} p<0.01 ;{ }^{* * *} p<0.001$.

Additional file 5: Fig. S4. NRPs KO in RENCA tumor cells inhibited experimental RCC growth in immunodeficient mice. (A) Experimental tumors in nude mice were obtained after injection of $3 \times 10^{5}$ control (Ctrl, 10 mice) or NRPs KO RENCA cells ( 5 mice for each condition). One NRP1 KO clone (4.1 7) and one NRP2 KO clone (5.1 8) were injected. Tumor volume at the indicated times is presented. Each curve stands for an individual mouse.

Additional file 6: Fig. S5. In-vivo effects of NRPa-308 on mice weight. The weight of nude mice xenografted with $786-\mathrm{O}$ cells and treated with increasing doses of NRPa-308 was evaluated once a week.

\section{Abbreviations}

ATCC: American type culture collection; BSA: Bovine serum albumin; cCRCC: Clear cell Renal Cell Carcinoma; CXCL: C-X-C motif chemokine; DNA: Deoxyribonucleic acid; EMT : Epithelial/mesenchymal transition; GFP : Green fluorescent protein; HDF : Normal dermal fibroblast; HGF : Hepatocyte growth factor; HIF : Hypoxia inducible factor; HRP : Horseradish peroxidase; $\mathrm{IC}_{50}$ : Half maximal inhibitory concentration; $\mathrm{KO}$ : Knock-out; Luc : Luciferase; mccRCC : Metastatic clear cell Renal Cell Carcinoma; MET : c-MET tyrosine-kinase receptor; mRNA : Messenger ribonucleic acid; NRP : Neuropilin; PBS : Phosphate buffered saline; PEI : Polyethylenimine; GPCR : Quantitative polymerase chain reaction; sgRNA : Single guide ribonucleic acid; shRNA: Short-hairpin ribonucleic acid; SI: Selectivity index; TBS : Trisbuffered saline; TCGA : The cancer genome atlas; TKi : Tyrosine-kinase inhibitor; VEGF : Vascular endothelial growth factor; VEGFR: Vascular endothelial growth factor receptor; VHL : Von-Hippel Lindau; aSMA: a smooth muscle actin

\section{Acknowledgements}

Not applicable.

\section{Authors' contributions}

Conception and design: LD, RG, GP. Development of methodology: AD, EB, $J D, W, A K P, Y L, C M, M P$. Acquisition of data: AD, EB, JD, W, AKP, YL, NL, MM, $C M, M P$, Analysis and interpretation of data: $A D, L D, R G, G P$. Writing, review: $A D, O H, C G, L D, R G, G P$. Administrative, technical, or material support: GP. Study supervision: RG, GP. The author(s) read and approved the final manuscript.

\section{Funding}

The authors acknowledge funding from Helsinn Company. This work was supported by The Centre Scientifique de Monaco, the Fondation de France, the French National Institute for Cancer Research (INCA, SUNITRES contract), the Agence Nationale de la Recherche (ANR), the Ligue Nationale contre le Cancer (Equipe labellisée 2019), the "Conseil Général des Alpes Maritimes", the association "Cordon de Vie" directed by Mrs. Fabienne Mourou, the Fondation François Xavier Mora and the Fondation Flavien.

\section{Availability of data and materials}

All data generated or analyzed during this study are included in this published article and its supplementary information files. Patient datasets are those of the TCGA and are publicly available.

\section{Ethics approval}

All animal procedures were performed according to the Monaco animal experimentation guidelines in strict accordance with the recommendations in the Guide for the Care and Use of Laboratory Animals. Our experiments were approved by our internal ethic committee.

\section{Consent for publication}

Not Applicable.

\section{Competing interests}

The authors have declared that no competing interest exists.

\section{Author details}

${ }^{1}$ Scientific Center of Monaco, Biomedical Department, 8 Quai Antoine ler, MC-98000 Monaco, Principality of Monaco. ${ }^{2}$ LIA ROPSE, Laboratoire International Associé Université Côte d'Azur - Centre Scientifique de Monaco, Nice, France. ${ }^{3}$ Université de Paris, CiTCoM, UMR 8038 CNRS, F-75006 Paris, France. ${ }^{4}$ Faculty of Chemistry, University of Warsaw, Pasteura 1, 02-093 Warsaw, Poland. ${ }^{5}$ INSERM UMR 1163, Laboratory of Cellular and Molecular Basis of Normal Hematopoiesis and Hematological Disorders: Therapeutical Implications, F-75015 Paris, France. ${ }^{6}$ Université de Paris, Imagine Institut, F-75015 Paris, France. ${ }^{7}$ Université de Paris, LCBPT, UMR8601 CNRS, UFR Biomédicale des Saints-Pères, F-75006 Paris, France. ${ }^{8}$ Laboratoire GBCM EA7528, Conservatoire National des Arts et Métiers, HESAM Université, 2 Rue Conté, 75003 Paris, France. ${ }^{9}$ Université Côte d'Azur, ICN, UMR 7272 CNRS, F-06108 Nice, France. ${ }^{10}$ University Cote d'Azur (UCA), Institute for research on cancer and aging of Nice, CNRS UMR 7284; INSERM U1081, Centre Antoine Lacassagne, Nice, France.

Received: 15 December 2020 Accepted: 7 January 2021

Published online: 18 January 2021

\section{References}

1. Kim E, Zschiedrich S. Renal cell carcinoma in von Hippel-Lindau disease-from tumor genetics to novel therapeutic strategies. Front Pediatr. 2018;6:16

2. Signoretti S, Flaifel A, Chen YB, Reuter VE. Renal Cell Carcinoma in the Era of Precision Medicine: From Molecular Pathology to Tissue-Based Biomarkers. J Clin Oncol. 2018;36(36):3553-9. https://doi.org/10.1200/JCO.2018.79.2259 .

3. Schodel J, Grampp S, Maher ER, Moch H, Ratcliffe PJ, Russo P, et al. Hypoxia, hypoxia-inducible transcription factors, and renal Cancer. Eur Urol. 2016;69: 646-57.

4. Niland S, Eble JA. Neuropilins in the context of tumor vasculature. Int J Mol Sci. 2019;20(3):639

5. Motzer RJ, Ravaud A, Patard JJ, Pandha HS, George DJ, Patel A, et al. Adjuvant Sunitinib for high-risk renal cell carcinoma after nephrectomy: subgroup analyses and updated overall survival results. Eur Urol. 2018;73: 62-8.

6. Gore ME, Szczylik C, Porta C, Bracarda S, Bjarnason GA, Oudard S, et al. Final results from the large sunitinib global expanded-access trial in metastatic renal cell carcinoma. Br J Cancer. 2015;113:12-9.

7. Dufies M, Giuliano S, Ambrosetti D, Claren A, Ndiaye PD, Mastri M, et al. Sunitinib stimulates expression of VEGFC by tumor cells and promotes Lymphangiogenesis in clear cell renal cell carcinomas. Cancer Res. 2017;77: $1212-26$.

8. Liu WQ, Lepelletier Y, Montes M, Borriello L, Jarray R, Grepin R, et al. NRPa308, a new neuropilin-1 antagonist, exerts in vitro anti-angiogenic and antiproliferative effects and in vivo anti-cancer effects in a mouse xenograft model. Cancer Lett. 2018;414:88-98.

9. Cao Y, Hoeppner LH, Bach S. E G, Guo Y, Wang E, et al. Neuropilin-2 promotes extravasation and metastasis by interacting with endothelial alpha5 integrin. Cancer Res. 2013;73:4579-90.

10. Cao Y, Wang L, Nandy D, Zhang Y, Basu A, Radisky D, et al. Neuropilin-1 upholds dedifferentiation and propagation phenotypes of renal cell carcinoma cells by activating Akt and sonic hedgehog axes. Cancer Res. 2008;68:8667-72.

11. Dumond A, Demange L, Pages G. Neuropilins: relevant therapeutic targets to improve the treatment of cancers. Med Sci (Paris). 2020:36:487-96.

12. Wild JR, Staton CA, Chapple K, Corfe BM. Neuropilins: expression and roles in the epithelium. Int J Exp Pathol. 2012;93:81-103.

13. Brachet E, Dumond A, Liu WQ, Fabre M, Selkti M, Raynaud F, et al. Synthesis, 3D-structure and stability analyses of NRPa-308, a new promising anticancer agent. Bioorg Med Chem Lett. 2019;29:126710.

14. Motzer RJ, Tannir NM, McDermott DF, Aren Frontera O, Melichar B, Choueiri TK, et al. Nivolumab plus Ipilimumab versus Sunitinib in advanced renal-cell carcinoma. N Engl J Med. 2018;378:1277-90.

15. Grepin R, Ambrosetti D, Marsaud A, Gastaud L, Amiel J, Pedeutour F, et al. The relevance of testing the efficacy of anti-angiogenesis treatments on cells derived from primary tumors: a new method for the personalized treatment of renal cell carcinoma. PLoS One. 2014;9:e89449. 
16. Grepin R, Guyot M, Jacquin M, Durivault J, Chamorey E, Sudaka A, et al. Acceleration of clear cell renal cell carcinoma growth in mice following bevacizumab/Avastin treatment: the role of CXCL cytokines. Oncogene. 2012:31:1683-94.

17. Berman HM, Westbrook J, Feng Z, Gilliland G, Bhat TN, Weissig H, et al. The Protein Data Bank. Nucleic Acids Res. 2000;28:235-42

18. Wolber G, Langer T. LigandScout: 3-D pharmacophores derived from protein-bound ligands and their use as virtual screening filters. J Chem Inf Model. 2005:45:160-9.

19. Trott O, Olson AJ. AutoDock Vina: improving the speed and accuracy of docking with a new scoring function, efficient optimization, and multithreading. J Comput Chem. 2010;31:455-61.

20. Grepin R, Guyot M, Giuliano S, Boncompagni M, Ambrosetti D, Chamorey E, et al. The CXCL7/CXCR1/2 axis is a key driver in the growth of clear cell renal cell carcinoma. Cancer Res. 2014;74:873-83.

21. Gao J, Aksoy BA, Dogrusoz U, Dresdner G, Gross B, Sumer SO, et al. Integrative analysis of complex cancer genomics and clinical profiles using the cBioPortal. Sci Signal. 2013;6:pl1.

22. Cerami E, Gao J, Dogrusoz U, Gross BE, Sumer SO, Aksoy BA, et al. The cBio cancer genomics portal: an open platform for exploring multidimensional cancer genomics data. Cancer Discov. 2012;2:401-4.

23. Napolitano V, Tamagnone L. Neuropilins controlling Cancer therapy responsiveness. Int J Mol Sci. 2019;20(8):2049. https://doi.org/10.3390/ ijms20082049.

24. Hsu PD, Scott DA, Weinstein JA, Ran FA, Konermann S, Agarwala V, et al. DNA targeting specificity of RNA-guided Cas9 nucleases. Nat Biotechnol. 2013;31:827-32.

25. Xu H, Xiao T, Chen CH, Li W, Meyer CA, Wu Q, et al. Sequence determinants of improved CRISPR sgRNA design. Genome Res. 2015;25:1147-57.

26. Calvo E, Porta C, Grunwald V, Escudier B. The current and evolving landscape of first-line treatments for advanced renal cell carcinoma. Oncologist. 2019;24:338-48.

27. Giuliano S, Cormerais Y, Dufies M, Grepin R, Colosetti P, Belaid A, et al. Resistance to sunitinib in renal clear cell carcinoma results from sequestration in lysosomes and inhibition of the autophagic flux. Autophagy. 2015;11:1891-904.

28. Giuliano S, Dufies M, Ndiaye PD, Viotti J, Borchiellini D, Parola J, et al. Resistance to lysosomotropic drugs used to treat kidney and breast cancers involves autophagy and inflammation and converges in inducing CXCL5. Theranostics. 2019;9:1181-99.

29. Cao Y. E G, Wang E, pal K, Dutta SK, Bar-Sagi D, et al. VEGF exerts an angiogenesis-independent function in cancer cells to promote their malignant progression. Cancer Res. 2012;72:3912-8.

30. Giuliano S, Pages G. Mechanisms of resistance to anti-angiogenesis therapies. Biochimie. 2013;95:1110-9.

31. Rini Bl, Plimack ER, Stus V, Gafanov R, Hawkins R, Nosov D, et al. Pembrolizumab plus Axitinib versus Sunitinib for advanced renal-cell carcinoma. N Engl J Med. 2019:380:1116-27.

32. Motzer RJ, Penkov K, Haanen J, Rini B, Albiges L, Campbell MT, et al. Avelumab plus Axitinib versus Sunitinib for advanced renal-cell carcinoma. N Engl J Med. 2019;380:1103-15.

33. Ravaud A, Motzer RJ, Pandha HS, George DJ, Pantuck AJ, Patel A, et al. Adjuvant Sunitinib in high-risk renal-cell carcinoma after nephrectomy. N Engl J Med. 2016;375:2246-54.

34. Haas NB, Manola J, Uzzo RG, Flaherty KT, Wood CG, Kane C, et al. Adjuvant sunitinib or sorafenib for high-risk, non-metastatic renal-cell carcinoma (ECOG-ACRIN E2805): a double-blind, placebo-controlled, randomised, phase 3 trial. Lancet. 2016;387:2008-16.

35. Hsieh JJ, Purdue MP, Signoretti S, Swanton C, Albiges L, Schmidinger M, et al. Renal cell carcinoma. Nat Rev Dis Primers. 2017;3:17009.

\section{Publisher's Note}

Springer Nature remains neutral with regard to jurisdictional claims in published maps and institutional affiliations.

Ready to submit your research? Choose BMC and benefit from:

- fast, convenient online submission

- thorough peer review by experienced researchers in your field

- rapid publication on acceptance

- support for research data, including large and complex data types

- gold Open Access which fosters wider collaboration and increased citations

- maximum visibility for your research: over $100 \mathrm{M}$ website views per year

At BMC, research is always in progress.

Learn more biomedcentral.com/submissions 